\title{
ON COMPUTING STABLE LAGRANGIAN SUBSPACES OF HAMILTONIAN MATRICES AND SYMPLECTIC PENCILS*
}

\author{
WEN-WEI LIN ${ }^{\dagger}$ AND CHERN-SHUH WANG ${ }^{\ddagger}$
}

\begin{abstract}
This paper presents algorithms for computing stable Lagrangian invariant subspaces of a Hamiltonian matrix and a symplectic pencil, respectively, having purely imaginary and unimodular eigenvalues. The problems often arise in solving continuous- or discrete-time $H^{\infty}$-optimal control, linear-quadratic control and filtering theory, etc. The main approach of our algorithms is to determine an isotropic Jordan subbasis corresponding to purely imaginary (unimodular) eigenvalues by using the associated Jordan basis of the square of the Hamiltonian matrix (the $S+S^{-1}$-transformation of the symplectic pencil). The algorithms preserve structures and are numerically efficient and reliable in that they employ only orthogonal transformations in the continuous case.
\end{abstract}

Key words. stable Lagrangian subspace, purely imaginary eigenvalue, Hamiltonian matrix, unimodular eigenvalue, symplectic pencil

AMS subject classifications. 47A15, 15A18, 15A21,, 15A22, 15A24

PII. S0895479894272712

1. Introduction. A matrix $M \in \mathbf{R}^{2 n \times 2 n}$ is said to be Hamiltonian if $J M=$ $(J M)^{T}$, where $J \equiv J_{n}=\left[\begin{array}{cc}O_{n} & I_{n} \\ -I_{n} & O_{n}\end{array}\right]$. Here $I_{n}$ is the $n \times n$ identity matrix and $O_{n}$ is the $n \times n$ zero matrix. A matrix $S \in \mathbf{R}^{2 n \times 2 n}$ is symplectic if $S^{T} J S=J$. A linear pencil $N-\lambda L$ with $N, L \in \mathbf{R}^{2 n \times 2 n}$ is said to be symplectic if $N J N^{T}=L J L^{T}$. If we partition a Hamiltonian matrix $M$ and a symplectic pencil $N-\lambda L$ comfortably with $J$, respectively, then we have

$$
M=\left[\begin{array}{cc}
A & G \\
H & -A^{T}
\end{array}\right], \quad G=G^{T}, \quad H=H^{T},
$$

and

$$
N=\left[\begin{array}{cc}
A & O \\
-H & I
\end{array}\right], \quad L=\left[\begin{array}{cc}
I & G \\
O & A^{T}
\end{array}\right], \quad G=G^{T}, \quad H=H^{T} .
$$

Our interest in the Hamiltonian matrix $M$ in (1.1) and the symplectic pencil $N-\lambda L$ in (1.2), respectively, stems from the fact that if

$$
\left[\begin{array}{cc}
A & G \\
H & -A^{T}
\end{array}\right]\left[\begin{array}{l}
\Omega_{1} \\
\Omega_{2}
\end{array}\right]=\left[\begin{array}{l}
\Omega_{1} \\
\Omega_{2}
\end{array}\right] W, \quad \Omega_{1}, \Omega_{2}, W \in \mathbf{R}^{n \times n}
$$

then $X=-\Omega_{2} \Omega_{1}^{-1}$ (if $\Omega_{1}^{-1}$ exists) solves the continuous-time algebraic Riccati equation (CARE)

$$
-X G X+X A+A^{T} X+H=0
$$

* Received by the editors August 10, 1994; accepted for publication (in revised form) by P. Van Dooren July 12, 1996.

http://www.siam.org/journals/simax/18-3/27271.html

† Institute of Applied Mathematics, Tsing Hua University, Hsinchu, Taiwan (wwlin@am.nthu. edu.tw).

$\ddagger$ Department of Applied Mathematics, Chiao Tung University, Hsinchu, Taiwan (d788101@am. nthu.edu.tw). 
and if

$$
\left[\begin{array}{cc}
A & O \\
-H & I
\end{array}\right]\left[\begin{array}{l}
\Omega_{1} \\
\Omega_{2}
\end{array}\right]=\left[\begin{array}{cc}
I & G \\
O & A^{T}
\end{array}\right]\left[\begin{array}{l}
\Omega_{1} \\
\Omega_{2}
\end{array}\right] W
$$

then $X=-\Omega_{2} \Omega_{1}^{-1}$ (if $\Omega_{1}^{-1}$ exists) solves the discrete-time algebraic Riccati equation (DARE)

$$
A^{T} X A-X-A^{T} X G(I+X G)^{-1} X A+H=0 .
$$

In fact, the Hamiltonian matrix and the symplectic pencil are often derived from continuous- and discrete-time optimal control problems, respectively, e.g., [5, 6, 8, $10,11,13,14]$. To obtain an optimizer, especially a stabilizing optimizer, of optimal control problems, one must compute a particular invariant subspace satisfying (1.3) or (1.5). This particular invariant subspace is usually referred to as a stable Lagrangian subspace.

Definition 1.1. A subspace $\mathcal{S} \subset \mathbf{R}^{2 n}$ is isotropic if

$$
x^{T} J y=0 \quad \text { for all } x, y \in \mathcal{S} .
$$

Definition 1.2. A subspace $\mathcal{Y} \subset \mathbf{R}^{2 n}$ is called an $M$-stable isotropic subspace if $\mathcal{Y}$ satisfies that (i) $M \mathcal{Y} \subset \mathcal{Y}$, (ii) $\mathcal{Y}$ is isotropic, and (iii) $\operatorname{Re}\left(\lambda\left(\left.M\right|_{\mathcal{Y}}\right)\right) \leq 0$. Here $\lambda\left(\left.M\right|_{\mathcal{Y}}\right)$ denotes an eigenvalue of $M$ restricted in $\mathcal{Y}$.

Definition 1.3. A subspace $\mathcal{W} \subset \mathbf{R}^{2 n}$ is called an $(N, L)$-stable isotropic subspace if (i) $\mathcal{W}$ is invariant under $(N, L)[25]$; i.e., there is a subspace $\mathcal{V}$ such that $N \mathcal{W}, L \mathcal{W} \subset \mathcal{V}$; (ii) $\mathcal{W}$ is isotropic; and (iii) $|\lambda((N, L) \mid \mathcal{W})| \leq 1$.

Definition 1.4. If $\mathcal{Y}_{\mathcal{L}} \subset \mathbf{R}^{2 n}$ is an $M$-stable isotropic subspace with $\operatorname{dim}\left(\mathcal{Y}_{\mathcal{L}}\right)=$ $n$, then $\mathcal{Y}_{\mathcal{L}}$ is called an $M$-stable Lagrangian subspace.

DeFINITION 1.5. If $\mathcal{W}_{\mathcal{L}} \subset \mathbf{R}^{2 n}$ is an $(N, L)$-stable isotropic subspace with $\operatorname{dim}\left(\mathcal{W}_{\mathcal{L}}\right)=n$, then $\mathcal{W}_{\mathcal{L}}$ is called an $(N, L)$-stable Lagrangian subspace.

For the continuous-time case, it is known that an $M$-stable Lagrangian subspace is closely related to an internally stabilizing controller of an $H^{\infty}$-control system $[5,8]$. In linear-quadratic control problems in which $(A, G)$ is stabilizable with $G$ positive semidefinite, we can obtain the unique "weak" stabilizing symmetric solution of CARE (1.4), and therefore an optimal controller by computing the unique $M$-stable Lagrangian subspace [14, 28]. In addition, several applications in Wiener filtering theory [26] and network synthesis [1] also need to compute an $M$-stable Lagrangian subspace. This is the reason why we are interested in computing an $M$-stable Lagrangian subspace. Unfortunately, an $M$-stable Lagrangian subspace does not always exist, while some nonzero purely imaginary eigenvalues of $M$ have odd partial multiplicities. A counterexample can be found in [21].

To guarantee the existence of an $M$-stable Lagrangian subspace, $M$ must satisfy the following assumption.

(A1) The partial multiplicities of all purely imaginary eigenvalues are all even.

If we require that

(R1) the $M$-stable Lagrangian subspace $\mathcal{Y}_{\mathcal{L}}$ have the lowest Jordan degree (that is, there is no other $M$-stable Lagrangian subspace having total Jordan degree smaller than that of $\mathcal{Y}_{\mathcal{L}}$ ), then the desired $M$-stable Lagrangian subspace $\mathcal{Y}_{\mathcal{L}}$ is unique determined.

We will discuss the details of this result in the next section. 
The first purpose of this paper is to propose an efficient, reliable, and structurepreserving algorithm for computing the $M$-stable Lagrangian subspace satisfying (R1) under the assumption (A1). For Hamiltonian matrices with purely imaginary eigenvalues, Clements and Glover [5] proposed an eigenvector deflation technique that guarantees that the eigenvalues appear with the correct pairing. This is certainly an advantage over the general $Q R$ or $Q Z$ method [12, 15,24], but this method still ignores the structure in part during the process. In another recent paper, Ammar and Mehrmann [2] proposed an elegant method, only using symplectic orthogonal transformations to compute the $M$-stable Lagrangian subspace. Combining the method with at least one step of defect correction is highly advisable. But, there are still numerical difficulties in convergence of deflation steps if purely imaginary eigenvalues occur [20, section 18, p. 143].

To avoid the numerical difficulties mentioned above, we shall develop a stable and structure-preserving algorithm as a preprocessing step to deflate all purely imaginary eigenvalues and to get a reduced Hamiltonian matrix having no purely imaginary eigenvalues. Then the rest of the $M$-stable Lagrangian subspace corresponding to stable eigenvalues with negative real parts can be computed by some reliable algorithms, such as in $[2,23,29]$. In our algorithm, we first compute the skew-Hamiltonian Schur decomposition of $M^{2}$ by using the numerically stable square reduced algorithm of Van Loan [27]. Then, we apply the algorithm proposed in [3] or [17] to the skewHamiltonian Schur matrix to determine the Jordan subbasis corresponding to the nonpositive eigenvalues of $M^{2}$. These algorithms are numerically reliable and need only $O\left(n^{2}\right)$ flops if the number of nonpositive eigenvalues of $M^{2}$ is of order $O(1)$. Based on elementary linear algebra theory, we can determine an associated Jordan subbasis $Y$ corresponding to purely imaginary eigenvalues of $M$ by using the Jordan subbasis corresponding to nonpositive eigenvalues of $M^{2}$. Under the assumption (A1) that each purely imaginary eigenvalue has even partial multiplicities, by applying an isotropicity requirement, we can separate an isotropic Jordan subbasis $\Upsilon$ corresponding to each first half of Jordan blocks of purely imaginary eigenvalues from $Y$. Indeed, the subspace $\operatorname{span}\{\Upsilon\}$ lies on the $M$-stable Lagrangian subspace. Consequently, we deflate the isotropic subbasis $\Upsilon$ from $M$ by using symplectic orthogonal transformations and get a reduced Hamiltonian matrix having no purely imaginary eigenvalues.

For the discrete-time case, an $(N, L)$-stable Lagrangian subspace also play an important role for $H^{\infty}$-optimal or linear-quadratic control problems. In linear-quadratic control problems in which $(A, G)$ is stabilizable with $G$ positive semidefinite, the unique "weak" stabilizing symmetric solution of DARE (1.6) can be obtained by computing the $(N, L)$-stable Lagrangian subspace [13]. For the $H^{\infty}$-control problem a detailed treatment of the suboptimal controller versus the $H^{\infty}$-optimal control is not available. The suboptimal case is treated in detail in $[10,11]$. Although a factorization theory similar to [5] has not been developed for the discrete-time case, we still consider computing the $(N, L)$-stable Lagrangian subspace of $N-\lambda L$ from a theoretical point of view. To ensure the existence and uniqueness of the desired $(N, L)$-stable Lagrangian subspace with lowest Jordan degree, a related assumption and requirement as in the continuous-time case are listed as follows.

(A2) The partial multiplicities of all unimodular eigenvalues of $N-\lambda L$ are even.

$(\mathrm{R} 2)$ The $(N, L)$-stable Lagrangian subspace $\mathcal{W}_{\mathcal{L}}$ has the lowest Jordan degree. (That is, there is no other $(N, L)$-stable Lagrangian subspace having total Jordan degree smaller than that of $\mathcal{W}_{\mathcal{L}}$.) 
As in a continuous-time case, we can also develop a reliable and structure-preserving algorithm as a preprocessing step to deflate all unimodular eigenvalues and get a reduced symplectic pencil having no unimodular eigenvalues. Then the rest of the $(N, L)$-stable Lagrangian subspace can be computed by algorithms of [19] or [29]. In our algorithm we consider the $S+S^{-1}$-transformation of the symplectic pencil $N-\lambda L$ [18], i.e.,

$$
\Gamma-\lambda \Delta \equiv\left[\left(N J L^{T}+L J N^{T}\right)-\lambda L J L^{T}\right] J^{T},
$$

and then we compute the skew-Hamiltonian Schur pencil form of $\Gamma-\lambda \Delta$ by using the numerically stable algorithm proposed in [22]. As in the continuous-time case, we first compute a Jordan subbasis corresponding to eigenvalues of $\Gamma-\lambda \Delta$ with magnitudes between -2 and 2 by algorithms of [3] or [17] and then use it to determine an isotropic Jordan subbasis corresponding to each first half of Jordan blocks of unimodular eigenvalues of $N-\lambda L$. Further, we deflate this subbasis of $N-\lambda L$ by symplectic transformations and get a reduced symplectic pencil having no unimodular eigenvalues.

For convenience, we list some notation which are adopted in this paper.

$Z_{p}$ denotes an orthonormal matrix which forms an orthonormal subbasis of $M^{2}$ corresponding to the zero eigenvalue with the Jordan degree of $p$; i.e., for any nonzero vector $v \in \operatorname{span}\left\{Z_{p}\right\}$,

$$
\left(M^{2}\right)^{p} v=0 \text { and }\left(M^{2}\right)^{p-1} \neq 0 .
$$

$\widetilde{Z}_{p}$ denotes the matrix $\left[Z_{1}, \ldots, Z_{p}\right]$.

$Y_{p}$ denotes an orthonormal matrix which forms an orthonormal subbasis of $M$ corresponding to the zero eigenvalue with the Jordan degree of $p$; i.e., for any nonzero vector $v \in \operatorname{span}\left\{Y_{p}\right\}$,

$$
M^{p} v=0 \text { and } M^{p-1} \neq 0 .
$$

$\widetilde{Y}_{p}$ denotes the matrix $\left[Y_{1}, \ldots, Y_{p}\right]$.

$\Upsilon_{s}$ denotes an orthonormal matrix which forms an orthonormal subbasis of the maximal $M$-stable isotropic subspace corresponding to each first half of Jordan blocks of zero eigenvalue.

$J^{(\ell)}(\lambda)$ denotes an $\ell \times \ell$ elementary Jordan matrix corresponding to $\lambda$; i.e.,

$$
J^{(\ell)}(\lambda)=\left[\begin{array}{cccc}
\lambda & 1 & & \\
& \ddots & \ddots & \\
& & & 1 \\
& & & \lambda
\end{array}\right]_{\ell \times \ell} .
$$

$\Lambda^{(\ell)}(0)$ denotes an $\ell \times \ell$ matrix with

$$
\Lambda^{(\ell)}(0)=\left[\begin{array}{ll}
O_{\ell-1} & \\
& \delta_{\ell}
\end{array}\right], \quad \delta_{\ell}=1 \text { or } 0 .
$$

$e_{j} \equiv e_{j}^{(n)}$ is the $j$ th column vector of $n \times n$ identity matrix $I_{n}$.

$\mathcal{N}(A)$ denotes the null space of matrix $A$.

All script (calligraphic) capital letters, e.g., $\mathcal{Y}, \mathcal{W}$, etc. denote vector subspaces. 
This paper is organized as follows. In section 2 we summarize some preliminary results. In sections 3 and 4 we develop numerically reliable algorithms to compute the desired isotropic subspaces of a Hamiltonian matrix and a symplectic pencil, respectively, corresponding to purely imaginary and unimodular eigenvalues. In section 5 , we show some numerical results to illustrate the numerical reliability of our algorithms.

2. Preliminary. In this section, we review some important properties of a real Hamiltonian matrix and a real symplectic pencil which have been developed and exploited for several years. First, we state a theorem of [16] which gives a canonical form of a Hamiltonian matrix.

Theorem 2.1 (see [16]). Let $M \in \mathbf{R}^{2 n \times 2 n}$ be a Hamiltonian matrix. Then there is a symplectic matrix $S \in \mathbf{R}^{2 n \times 2 n}$ such that

$$
S^{-1} M S=\left[\begin{array}{cc}
\operatorname{diag}\left\{J_{0}, 0, T_{1}, J_{\nu}^{T}\right\} & \operatorname{diag}\left\{\Lambda_{0}, E_{\mu}, T_{2}, D_{\nu}\right\} \\
\operatorname{diag}\left\{0, E_{-\mu}, 0,-D_{\nu}\right\} & \operatorname{diag}\left\{-J_{0}^{T}, 0,-T_{1}^{T},-J_{\nu}\right\}
\end{array}\right]
$$

where $\mu=\left(\mu_{1}, \ldots, \mu_{k_{2}}\right)^{T} \in \mathbf{R}^{k_{2}}, T_{1} \in \mathbf{R}^{k_{3} \times k_{3}}$ with $\operatorname{Re}\left(\lambda\left(T_{1}\right)\right)<0, \nu=\left(\nu_{1}, \ldots, \nu_{k_{4}}\right)^{T} \in$ $\mathbf{R}^{k_{4}}$, and

$$
\begin{aligned}
J_{0} & =\operatorname{diag}\left\{J^{\left(m_{1}\right)}(0), \ldots, J^{\left(m_{k_{1}}\right)}(0)\right\}, \\
\Lambda_{0} & =\operatorname{diag}\left\{\Lambda^{\left(m_{1}\right)}(0), \ldots, \Lambda^{\left(m_{k_{1}}\right)}(0)\right\}, \\
E_{\mu} & =\operatorname{diag}\left\{E^{\left(n_{1}\right)}\left(\mu_{1}\right), \ldots, E^{\left(n_{k_{2}}\right)}\left(\mu_{k_{2}}\right)\right\}, \\
E_{-\mu} & =\operatorname{diag}\left\{E^{\left(n_{1}\right)}\left(-\mu_{1}\right), \ldots, E^{\left(n_{k_{2}}\right)}\left(-\mu_{k_{2}}\right)\right\}
\end{aligned}
$$

with ( $n_{j}$ an even integer),

$$
\begin{aligned}
& E^{\left(n_{j}\right)}\left(\mu_{j}\right)=\left[\begin{array}{ccccc}
0 & & & & \mu_{j} \\
& & & \mu_{j} & 1 \\
& & \cdot & -1 & \\
& \mu_{j} & \cdot & & \\
\mu_{j} & 1 & & & 0
\end{array}\right]_{n_{j} \times n_{j}}, \\
& E^{\left(n_{j}\right)}\left(-\mu_{j}\right)=\left[\begin{array}{ccccc}
0 & & & -1 & -\mu_{j} \\
& & \cdot & -\mu_{j} & \\
-1 & -\mu_{j} & & & \\
-\mu_{j} & & & 0
\end{array}\right]_{n_{j} \times n_{j}} \\
& J_{\nu}=\operatorname{diag}\left\{J^{\left(\ell_{1}\right)}(0), \ldots, J^{\left(\ell_{k_{4}}\right)}(0)\right\}, \\
& D_{\nu}=\operatorname{diag}\left\{D^{\left(\ell_{1}\right)}\left(\nu_{1}\right), \ldots, D^{\left(\ell_{k_{4}}\right)}\left(\nu_{k_{4}}\right)\right\}
\end{aligned}
$$

with $\left(\ell_{j}\right.$ an odd integer $)$

$$
D^{\left(\ell_{j}\right)}\left(\nu_{j}\right)=\left[\begin{array}{cccc}
0 & & & -\nu_{j} \\
& & \nu_{j} & \\
-\nu_{j} & & & 0
\end{array}\right]_{\ell_{j} \times \ell_{j}}
$$


and

$$
n=\sum_{j=1}^{k_{1}} m_{j}+\sum_{j=1}^{k_{2}} n_{j}+k_{3}+\sum_{j=1}^{k_{4}} \ell_{j} .
$$

By Theorem 2.1, we see that the Hamiltonian matrix $M$ contains zero eigenvalues and purely imaginary eigenvalues $\pm i \mu_{j}$ for $j=1, \ldots, k_{2}$ and $\pm i \nu_{j}$ for $j=1, \ldots, k_{4}$.

Under assumption (A1), the canonical form (2.1) becomes a simpler form,

$$
S^{-1} M S=\left[\begin{array}{cc}
\operatorname{diag}\left\{J_{0}, 0, T_{1}\right\} & \operatorname{diag}\left\{\Lambda_{0}, E_{\mu}, T_{2}\right\} \\
\operatorname{diag}\left\{0, E_{-\mu}, 0\right\} & \operatorname{diag}\left\{-J_{0}^{T}, 0,-T_{1}^{T},\right\}
\end{array}\right],
$$

where $\mu, T_{1}, J_{0}, \Lambda_{0}, E_{\mu}, E_{-\mu}$ are given in (2.1) with $n=\sum_{j=1}^{k_{1}} m_{j}+\sum_{j=1}^{k_{2}} n_{j}+k_{3}$.

Partition the symplectic matrix $S=\left[S_{1}, S_{2}, S_{3}, \widehat{S}_{1}, \widehat{S}_{2}, \widehat{S}_{3}\right]$ with the block type (2.2). Furthermore, we partition

$$
S_{1}=\left[S_{1}^{(1)}, \ldots, S_{1}^{\left(k_{1}\right)}\right] \text { and } \widehat{S}_{1}=\left[\widehat{S}_{1}^{(1)}, \ldots, \widehat{S}_{1}^{\left(k_{1}\right)}\right]
$$

comfortably with block type of $J_{0}$ and write $S_{1}^{(j)}$ and $\widehat{S}_{1}^{(j)}$ in the column vector forms

$$
S_{1}^{(j)}=\left[s_{1}^{(1, j)}, \ldots, s_{m_{j}}^{(1, j)}\right] \text { and } \widehat{S}_{1}^{(j)}=\left[\hat{s}_{1}^{(1, j)}, \ldots, \hat{s}_{m_{j}}^{(1, j)}\right]
$$

for $j=1, \ldots, k_{1}$. If $\delta_{j}=1$ (the $\left(m_{j}, m_{j}\right)$ th element of $\left.\Lambda^{\left(m_{j}\right)}(0)\right)$ for some $j \in$ $\left\{1, \ldots, k_{1}\right\}$, then the maximal $M$-stable isotropic subspace with lowest Jordan degree of $\operatorname{span}\left\{S_{1}^{(j)}, \widehat{S}_{1}^{(j)}\right\}$ is

$$
\mathcal{S}_{1}^{(j)}=\operatorname{span}\left\{S_{1}^{(j)}\right\}
$$

If $\delta_{j}=0$ for some $j \in\left\{1, \ldots, k_{1}\right\}$ (here $m_{j}$ must be even), then the maximal $M$-stable isotropic subspace with lowest Jordan degree of $\operatorname{span}\left\{S_{1}^{(j)}, \widehat{S}_{1}^{(j)}\right\}$ is

$$
\mathcal{S}_{1}^{(j)}=\operatorname{span}\left\{s_{1}^{(1, j)}, \ldots, s_{m_{j} / 2}^{(1, j)}, \hat{s}_{m_{j} / 2}^{(1, j)}, \ldots, \hat{s}_{m_{j}}^{(1, j)}\right\} .
$$

Partition

$$
S_{2}=\left[S_{2}^{(1)}, \ldots, S_{2}^{\left(k_{2}\right)}\right] \text { and } \widehat{S}_{2}=\left[\widehat{S}_{2}^{(1)}, \ldots, \widehat{S}_{2}^{\left(k_{2}\right)}\right]
$$

with the block type $E_{\mu}$ and write $S_{2}^{(j)}$ and $\widehat{S}_{2}^{(j)}$ in the column vector forms

$$
S_{2}^{(j)}=\left[s_{1}^{(2, j)}, \ldots, s_{n_{j}}^{(2, j)}\right] \text { and } \widehat{S}_{2}^{(j)}=\left[\hat{s}_{1}^{(2, j)}, \ldots, \hat{s}_{n_{j}}^{(2, j)}\right]
$$

for $j=1, \ldots, k_{2}$. The maximal $M$-stable isotropic subspace with lowest Jordan degree of $\operatorname{span}\left\{S_{2}^{(j)}, \widehat{S}_{2}^{(j)}\right\}$ is

$$
\mathcal{S}_{2}^{(j)}=\operatorname{span}\left\{s_{n_{j} / 2}^{(2, j)}, \ldots, s_{n_{j}}^{(2, j)}, \hat{s}_{1}^{(2, j)}, \ldots, \hat{s}_{n_{j} / 2}^{(2, j)}\right\} .
$$

Let $\mathcal{S}_{3} \equiv \operatorname{span}\left\{S_{3}\right\}$ denote a maximal $M$-stable isotropic subspace of $\operatorname{span}\left\{S_{3}, \widehat{S}_{3}\right\}$. Since $\mathcal{S}_{1}^{(j)}, j=1, \ldots, k_{1}, \mathcal{S}_{2}^{(j)}, j=1, \ldots, k_{2}$, and $\mathcal{S}_{3}$ are uniquely determined with lowest Jordan degree by collecting these $M$-stable isotropic subspaces and letting

$$
\mathcal{Y}_{\mathcal{L}}=\left(\bigoplus_{j=1}^{k_{1}} \mathcal{S}_{1}^{(j)}\right) \oplus\left(\bigoplus_{j=1}^{k_{2}} \mathcal{S}_{2}^{(j)}\right) \oplus \mathcal{S}_{3}
$$


we get that $\mathcal{Y}_{\mathcal{L}}$ is the $M$-stable Lagrangian subspace satisfying (R1).

From the above discussion, we see that the desired Lagrangian subspace $\mathcal{Y}_{\mathcal{L}}$ is spanned by the Jordan vectors corresponding to each first half of Jordan blocks of purely imaginary eigenvalue and the Jordan vectors corresponding to eigenvalues with negative real parts.

Assumption (A1) is necessary for the uniqueness of (R1). If we relax (A1) in that some partial multiplicities of zero eigenvalues of $M$ are permitted to be odd, then the $M$-stable Lagrangian subspace still exists, but the uniqueness of (R1) does not hold. For example, let $M=\operatorname{diag}\left\{J^{(3)}(0),-J^{(3)}(0)^{T}\right\}$. Then $M$ has zero eigenvalue with partial multiplicities 3,3 . It is easily seen that $\left\{e_{1}, e_{6}, e_{5}\right\},\left\{e_{1}, e_{2}, e_{6}\right\},\left\{e_{6}, e_{5}, e_{4}\right\}$, and $\left\{e_{1}, e_{2}, e_{3}\right\}$ are four distinct $M$-stable Lagrangian subspaces, but the first two have the same lowest Jordan degrees. As mentioned in section 1, if some nonzero eigenvalue has odd partial multiplicities, then the existence of $M$-stable Lagrangian subspace can fail. Let $M=\left[\begin{array}{cc}0 & 1 \\ -1 & 0\end{array}\right]$. Then $M$ has eigenvalues $\pm i$ associated with eigenvectors $\left[\begin{array}{l}1 \\ i\end{array}\right]$ and $\left[\begin{array}{c}1 \\ -i\end{array}\right]$, respectively. It is easy to verify that $M$ has no $M$-stable Lagrangian subspace.

The following theorem of [14] states an important result from linear-quadratic control problems.

THEOREM 2.2. Let $M$ be a Hamiltonian matrix as in (1.1). Let $G$ be positive semidefinite and $(A, G)$ be stabilizable. Assume (A1) holds. Then there exists a symplectic matrix $S$ such that $\Lambda^{\left(m_{j}\right)}(0)$ in $(2.2)$ has zeros everywhere except one in the $\left(m_{j}, m_{j}\right)$ th entry. Furthermore,

(i) there exists a unique $M$-stable Lagrangian subspace $\mathcal{Y}_{\mathcal{L}}$,

(ii) there exists a unique symmetric solution $X \in \mathbf{R}^{n \times n}$ of $C A R E$ in (1.4) such that $\operatorname{Re}(\lambda(A+G X)) \leq 0$ and $\operatorname{span}\left\{\left[\begin{array}{l}I \\ X\end{array}\right]\right\}=\mathcal{Y}_{\mathcal{L}}$.

Remark. For the case of Theorem 2.2, the only possible $M$-stable isotropic subspace corresponding to zero eigenvalues must have the form (2.3). The $M$-stable Lagrangian subspace $\mathcal{Y}_{\mathcal{L}}$ is then uniquely determined. Thus, requirement (R1) for $\mathcal{Y}_{\mathcal{L}}$ here is automatically satisfied.

For the symplectic pencil $N-\lambda L$, we want to find the $(N, L)$-stable Lagrangian subspace $\mathcal{W}_{\mathcal{L}}$. By a skillful transformation of $[20$, p. 120], we can deflate zero and infinity eigenvalues of $N-\lambda L$ simultaneously and obtain a reduced symplectic pencil $\widehat{N}-\lambda \widehat{L}$ having only nonzero finite eigenvalues. Thus, computing the $(N, L)$-stable Lagrangian subspace is equivalent to computing the stable Lagrangian subspace of the symplectic matrix $B=\widehat{L}^{-1} \widehat{N}$. It is easily seen that the Cayley transformation matrix

$$
M=(I+B)(I-B)^{-1}
$$

is Hamiltonian. Furthermore, since the transformation (2.5) is rational and $M, B$ are commuted, an $M$-stable Lagrangian subspace must be a stable Lagrangian subspace of $B$. Similar to the continuous-time case, we can conclude that the $(N, L)$-stable Lagrangian subspace $\mathcal{W}_{\mathcal{L}}$ is unique determined if (A2) and (R2) are satisfied.

Hereafter, for brevity, $M$-stable and $(N, L)$-stable Lagrangian subspaces mean the $M$-stable and the $(N, L)$-stable Lagrangian subspaces with lowest Jordan degrees, respectively.

3. Computing the stable Lagrangian subspace of a Hamiltonian matrix having purely imaginary eigenvalues. Let $M$ be the Hamiltonian matrix as in (1.1). Assume (A1) holds; i.e., the partial multiplicities of purely imaginary eigenvalues of $M$ are all even. In this section, we shall develop a reliable algorithm to compute 
the $M$-stable isotropic subspace $\mathcal{Y}$ corresponding to each first half of Jordan blocks of all purely imaginary eigenvalues and get a reduced Hamiltonian matrix having no purely imaginary eigenvalue. Combining $\mathcal{Y}$ with the isotropic subspace corresponding to the strictly stable eigenvalues of $M$, we obtain the desired $M$-stable Lagrangian subspace $\mathcal{Y}_{\mathcal{L}}$.

The main idea of our algorithm to determine $\mathcal{Y}$ is that we first compute a Jordan basis corresponding to nonpositive eigenvalues of $M^{2}$ and then use it to determine a Jordan basis corresponding to purely imaginary eigenvalues of $M$ and to determine an isotropic basis $\Upsilon$ of $\mathcal{Y}$.

We now consider the case of nonzero purely imaginary eigenvalues. Assume that the conjugate eigenvalue pair $\pm i \omega$ of $M$ have the Jordan blocks $\left\{J^{\left(2 m_{1}\right)}(i \omega), \ldots\right.$, $\left.J^{\left(2 m_{k}\right)}(i \omega)\right\}$ and $\left\{J^{\left(2 m_{1}\right)}(-i \omega), \ldots, J^{\left(2 m_{k}\right)}(-i \omega)\right\}$ with even orders, respectively. It is easily seen that the negative eigenvalue $-\omega^{2}$ of $M^{2}$ has the Jordan blocks $\left\{J^{\left(2 m_{j}\right)}\left(-\omega^{2}\right)\right.$, $\left.J^{\left(2 m_{j}\right)}\left(-\omega^{2}\right)\right\}_{j=1}^{k}$. Hence, the eigenspace of $M$ corresponding to each first half of Jordan blocks $\left\{J^{\left(m_{j}\right)}( \pm i \omega)\right\}_{j=1}^{k}$ is just the eigenspace of $M^{2}$ corresponding to each first half of Jordan blocks $\left\{J^{\left(m_{j}\right)}\left(-\omega^{2}\right), J^{\left(m_{j}\right)}\left(-\omega^{2}\right)\right\}_{j=1}^{k}$. Thus, the desired $M$-stable isotropic subspace can be determined directly from the associated eigenspace of $M^{2}$. The case of zero purely imaginary eigenvalue is more complicated than the case of nonzero purely imaginary eigenvalue. In the following we shall discuss this case carefully.

Let $\left\{2 m_{1}, \ldots, 2 m_{k}\right\}$ with $m_{1} \leq \cdots \leq m_{k}$ be the partial multiplicities of the zero eigenvalues of $M$ and $n_{0}=2 \sum_{j=1}^{k} m_{j}$ be the algebraic multiplicity of zero eigenvalues. Let

$$
Y=\left[Y_{1}^{(0)}, \ldots, Y_{2 m_{k}}^{(k-1)}\right]
$$

be an orthonormal basis of the subspace spanned by the associated Jordan vectors, where the submatrix $Y_{p}^{(j)}$ for $p=1, \ldots, 2 m_{k}$ is a $2 n \times(k-j)$ orthonormal matrix of degree $p$ and $j \equiv j(p) \in\{0, \ldots, k-1\}$ is an integer function in $p$ such that

$$
2 m_{j}<p \leq 2 m_{j+1}\left(m_{0}=0\right) .
$$

Remark. (i) A matrix $Y_{p}$ is of degree $p$ if any nonzero vector $v \in \operatorname{span}\left\{Y_{p}\right\}$ satisfies $M^{p} v=0$ and $M^{p-1} v \neq 0$. (ii) Since the mutually orthogonal subspaces spanned by $\left\{Y_{p}^{(j)}\right\}$ are unique $\left(p=1, \ldots, 2 m_{k}\right)$, for convenience we identify any two orthonormal bases of $\operatorname{span}\left\{Y_{p}^{(j)}\right\}$.

Furthermore, we define

$$
\tilde{Y}_{p}^{(j)}=\left[Y_{1}^{(0)}, \ldots, Y_{p}^{(j)}\right]
$$

as the submatrix of $Y$ of degree less than or equal to $p$. From elementary algebra theory, we see that the partial multiplicities of zero eigenvalues of $M^{2}$ are $\left\{m_{1}, m_{1}, \ldots, m_{k}, m_{k}\right\}$. Let

$$
Z=\left[Z_{1}^{(0)}, \ldots, Z_{m_{k}}^{(k-1)}\right]
$$

be an orthonormal basis of the associated Jordan vectors, where the submatrix $Z_{p}^{(j)}$ for $p=1, \ldots, m_{k}$ is a $2 n \times 2(k-j)$ orthonormal matrix of degree $p$ and $j \equiv j(p) \in$ $\{0, \ldots, k-1\}$ is an integer function in $p$ such that

$$
m_{j}<p \leq m_{j+1}\left(m_{0}=0\right) .
$$


We also define

$$
\widetilde{Z}_{p}^{(j)}=\left[Z_{1}^{(0)}, \ldots, Z_{p}^{(j)}\right]
$$

as the submatrix of $Z$ of degree less than or equal to $p$. Let $\Upsilon_{s}$ be an orthonormal isotropic subbasis corresponding to each first half of Jordan blocks of zero eigenvalues. In fact, $\Upsilon_{s}$ here is an orthonormal basis of the maximal isotropic subspace corresponding to zero eigenvalues and $\operatorname{span}\left\{\Upsilon_{s}\right\} \subset \mathcal{Y}_{\mathcal{L}}$. The approach of our algorithm is that we use $Z$ to determine $Y$ and then use $Y$ to compute $\Upsilon_{s}$.

We now develop a reliable algorithm to compute the matrix $Z$ described in $(3.3 \mathrm{a}, \mathrm{b})$. For convenience hereafter, we assume that the only purely imaginary eigenvalue of $M$ is zero.

ALGORITHM 3.1. This algorithm computes an orthonormal subbasis $Z=\left[Z_{1}^{(0)}, \ldots\right.$, $\left.Z_{m_{k}}^{(k-1)}\right]$ of $M^{2}$ corresponding to zero eigenvalues.

Step 1: Reduce $M^{2}$ to a Hessenberg matrix by using the squared reduced algorithm of [27]. That is, find a $2 n \times 2 n$ symplectic orthogonal matrix $Q$ so that

$$
Q^{T} M^{2} Q=H \equiv\left[\begin{array}{cc}
H_{1} & K_{1} \\
O & H_{1}^{T}
\end{array}\right],
$$

where $H_{1}$ is upper Hessenberg and $K_{1}$ is skew-symmetric.

Step 2: Reduce $H_{1}$ to a real Schur form by the $Q R$ algorithm, e.g., [9, p. 228]. That is, find an $n \times n$ orthogonal matrix $Q_{1}$ so that

$$
Q_{1}^{T} H_{1} Q_{1}=R_{1}, \quad Q_{1}^{T} K_{1} Q_{1}=S_{1},
$$

where $R_{1}$ is quasi-upper triangular.

Let $n_{0}=$ the algebraic multiplicity of zero eigenvalues of $M^{2}$.

Let

$$
\begin{aligned}
& H:=\left[\begin{array}{cc}
I & O \\
O & \hat{I}
\end{array}\right]\left[\begin{array}{cc}
R_{1} & S_{1} \\
O & R_{1}^{T}
\end{array}\right]\left[\begin{array}{cc}
I & O \\
O & \hat{I}
\end{array}\right] \text { (quasi-upper triangular), } \\
& Q:=\left[\begin{array}{cc}
Q_{1} & O \\
O & Q_{1}
\end{array}\right]\left[\begin{array}{cc}
I & O \\
O & \hat{I}
\end{array}\right] \text {, where } \hat{I}=\left[\begin{array}{ccc}
0 & & 1 \\
& & \cdot \\
& \cdot & \\
1 & & 0
\end{array}\right] \text {. }
\end{aligned}
$$

Set $E:=I_{2 n}, j=0, q=1$, and $m_{0}=0$.

\section{Step 3: Repeat:}

3.1 Find an orthonormal basis $\widehat{B}_{0}$ of null space of $H$ by applying an $R R Q R$ factorization of [4]. That is, find a permutation $\Pi_{1}$ and an orthogonal matrix $V_{1}$ such that

$$
\Pi_{1} H V_{1}=\left(\begin{array}{cc}
O & X \\
O & \widehat{H}
\end{array}\right)
$$

where $\widehat{H}$ is quasi-upper triangular. Let $\gamma_{H}$ be the nullity of $H$.

Set $\widehat{B}_{0}=V_{1}\left[\begin{array}{c}I_{\gamma_{H}} \\ 0\end{array}\right]$.

Comment: An RRQR factorization of a quasi-upper triangular $H$ needs only $O\left(n^{2}\right)$ flops if $n_{0} \ll n$. 
- If $q=1$, then

$$
k=\frac{\gamma_{H}}{2}, \quad \gamma^{*}=\gamma_{H}, \quad \text { Jump }=0, \quad B_{0}=\widehat{B}_{0},
$$

else

$$
\gamma=\gamma_{H}, \quad J u m p=\gamma^{*}-\gamma, \quad B_{0}=\left[\begin{array}{c}
0 \\
\widehat{B}_{0}
\end{array}\right] \in \mathbf{R}^{2 n \times \gamma_{H}}
$$

- If Jump $\neq 0$, then for $\ell=j+1, \ldots, j+\frac{\text { Jump }}{2}$, set $m_{\ell}=q-1$ and update $j=j+\frac{\text { Jump }}{2}, \gamma^{*}=\gamma$.

- Set $Z_{q}^{(j)}=Q B_{0}$.

- If $j=k$, then stop.

3.2 Find two orthogonal matrices $U_{2}$ and $V_{2}$ by using Algorithm 3.1.1 proposed by Beelen and Van Dooren [3] such that

$$
U_{2}^{T}\left(\Pi_{1} H V_{1}\right) V_{2}=\left[\begin{array}{ll}
0 & H_{12} \\
0 & H_{22}
\end{array}\right], \quad U_{2}^{T}\left(\Pi_{1} E V_{1}\right) V_{2}=\left[\begin{array}{cc}
E_{11} & E_{12} \\
0 & E_{22}
\end{array}\right] .
$$

Comment: (i) Here the matrix $\mathrm{H}_{22}$ is preserved to be quasi-upper triangular and $E_{11}$ is nonsingular. Algorithm 3.1.1 of [3] used in Step 3.2 needs only $O\left(n^{2}\right)$ flops. (ii) This substep determines the partial multiplicities and an orthonormal basis for the associated Jordan vectors [3].

3.3 Update (deflation step):

- $H:=H_{22}$ (dimension reduced).

- $E:=E_{22}$ (dimension reduced).

- If $q=1$, then set $Q=Q\left(V_{1} V_{2}\right)$, else set $Q=Q\left[\begin{array}{cc}I & 0 \\ 0 & V_{1} V_{2}\end{array}\right] \in \mathbf{R}^{2 n \times 2 n}$.

- Set $q=q+1$, go to Repeat.

Remark. (i) Instead of Step 3.2, one can also use a nonequivalence transformation to deflate the zero eigenvalues of the pencil $H-\lambda E$ [17]. The algorithm uses nonunitary transformations but needs only about one-fourth flops of Algorithm 3.1.1 of [3]. (ii) If $M^{2}$ has a negative eigenvalue $-\omega^{2}$, then we replace the matrix $H$ in Step 3 by $H+\omega^{2} I$ and perform the same process to compute an associated Jordan basis corresponding to $-\omega^{2}$. (iii) This algorithm uses only orthogonal transformations. The accuracy of the computed orthonormal Jordan subbasis $Z$ depends on the sensitivity of the computed nonpositive eigenvalues $-\omega^{2}$ of $M^{2}$. It is shown in [27] that the computed $\pm i \omega$ are the exact eigenvalues of a matrix $M+E$ where $\|E\|$ depends on the square root of the machine precision. Hence, the accuracy of the computed $Z$ is reliable when the sensitivity of $\pm i \omega$ of $M$ is acceptable.

The following theorem gives the relation between orthonormal Jordan bases corresponding to zero eigenvalues of $M^{2}$ and $M$, respectively. We use the notation defined in (3.1)-(3.4) but omit the superscript $(j)$.

Let $\widetilde{Z}=\left[Z_{1}, \ldots, Z_{q}\right]$ and $\widetilde{Y}_{p}=\left[Y_{1}, \ldots, Y_{p}\right]$, where $Z_{q}$ and $Y_{p}$ are orthonormal Jordan bases of $M^{2}$ and $M$, respectively, of degree $q$ and $p$ for $q=1, \ldots, m_{k}$ and $p=1, \ldots, 2 m_{k}$.

TheOREM 3.2. For $p=1, \ldots, m_{k}$, it holds that

(i) $\operatorname{span}\left\{\widetilde{Y}_{2 p}\right\}=\operatorname{span}\left\{\widetilde{Z}_{p}\right\}$,

(ii) $\operatorname{span}\left\{Z_{p}\right\}=\operatorname{span}\left\{Y_{2 p-1}\right\} \oplus \operatorname{span}\left\{Y_{2 p}\right\}$, 
(iii) $\operatorname{span}\left\{Y_{2 p-1}\right\}=\operatorname{span}\left\{\left(I-\widetilde{Z}_{p-1} \widetilde{Z}_{p-1}^{T}\right) M Z_{p}\right\}=\operatorname{span}\left\{\left(Z_{p} Z_{p}^{T}\right) M Z_{p}\right\}$,

(iv) if $W_{2 p-1}$ is an orthonormal basis of $\operatorname{span}\left\{\left(I-\widetilde{Z}_{p-1} \widetilde{Z}_{p-1}^{T}\right) M Z_{p}\right\}$, then $\operatorname{span}\left\{Y_{2 p}\right\}=\operatorname{span}\left\{\left(I-W_{2 p-1} W_{2 p-1}^{T}\right) Z_{p}\right\}$.

For convenience, here we use $\widetilde{Z}_{0}=0$.

Proof. (i) Since $\left(M^{2}\right)^{p} v=M^{2 p} v$ for any $v \in \mathbf{R}^{2 n \times 1}$, (i) follows.

(ii) From (i), we have

$$
\operatorname{span}\left\{Y_{2 p}\right\} \oplus \operatorname{span}\left\{Y_{2 p-1}\right\} \oplus \operatorname{span}\left\{\widetilde{Y}_{2 p-2}\right\}=\operatorname{span}\left\{Z_{p}\right\} \oplus \operatorname{span}\left\{\widetilde{Z}_{p-1}\right\} .
$$

Furthermore, both subspaces $\operatorname{span}\left\{Z_{p}\right\}$ and $\operatorname{span}\left\{Y_{2 p}\right\} \oplus \operatorname{span}\left\{Y_{2 p-1}\right\}$ are orthogonal to $\operatorname{span}\left\{\widetilde{Y}_{2 p-2}\right\}$ (i.e., $\operatorname{span}\left\{\widetilde{Z}_{p-1}\right\}$ ). Hence, (ii) is proved.

(iii) By the definition of $Z_{p}$, we have

$$
\operatorname{span}\left\{M Z_{p}\right\} \subset \operatorname{span}\left\{\widetilde{Y}_{2 p-1}\right\}=\operatorname{span}\left\{Y_{2 p-1}\right\} \oplus \operatorname{span}\left\{\widetilde{Z}_{p-1}\right\} .
$$

This implies that

$$
\operatorname{span}\left\{\left(I-\widetilde{Z}_{p-1} \widetilde{Z}_{p-1}^{T}\right) M Z_{p}\right\} \subset \operatorname{span}\left\{Y_{2 p-1}\right\} .
$$

On the other hand, from (ii), we have

$$
\operatorname{span}\left\{\left(I-\widetilde{Z}_{p-1} \widetilde{Z}_{p-1}^{T}\right) M Y_{2 p}\right\} \subset \operatorname{span}\left\{\left(I-\widetilde{Z}_{p-1} \widetilde{Z}_{p-1}^{T}\right) M Z_{p}\right\} .
$$

By (3.6) and (3.7), it is easily seen that

$$
\begin{aligned}
\operatorname{dim}\left(\operatorname{span}\left\{\left(I-\widetilde{Z}_{p-1} \widetilde{Z}_{p-1}^{T}\right) M Y_{2 p}\right\}\right) & \leq \operatorname{dim}\left(\operatorname{span}\left\{\left(I-\widetilde{Z}_{p-1} \widetilde{Z}_{p-1}^{T}\right) M Z_{p}\right\}\right) \\
& \leq \operatorname{dim}\left(\operatorname{span}\left\{Y_{2 p-1}\right\}\right) .
\end{aligned}
$$

From (3.6) and (3.7), it follows that to verify the first equality of (iii) it is sufficient to show that both inequalities in (3.8) hold. Now, suppose that

$$
\operatorname{dim}\left(\operatorname{span}\left\{\left(I-\widetilde{Z}_{p-1} \widetilde{Z}_{p-1}^{T}\right) M Y_{2 p}\right\}\right)<\operatorname{dim}\left(\operatorname{span}\left\{Y_{2 p-1}\right\}\right) .
$$

Since all partial multiplicities of zero eigenvalues are even,

$$
\operatorname{dim}\left(\operatorname{span}\left\{Y_{2 p}\right\}\right)=\operatorname{dim}\left(\operatorname{span}\left\{Y_{2 p-1}\right\}\right) .
$$

From (3.9) and (3.10) it follows that the column vectors of $\left(I-\widetilde{Z}_{p-1} \widetilde{Z}_{p-1}^{T}\right) M Y_{2 p}$ are linearly dependent. Thus, there exists a nonzero vector $\xi$ such that

$$
\left(I-\widetilde{Z}_{p-1} \widetilde{Z}_{p-1}^{T}\right) M Y_{2 p} \xi=0 .
$$

This implies that $M Y_{2 p} \xi \in \operatorname{span}\left\{\widetilde{Z}_{p-1}\right\}$. By the definition of $\widetilde{Z}_{p-1}$, we then have

$$
M^{2 p-1} Y_{2 p} \xi=\left(M^{2}\right)^{p-1} M Y_{2 p} \xi=0 .
$$

This contradicts the definition of $Y_{2 p}$. Therefore, the strict inequality in (3.9) does not hold; i.e., both equalities in (3.8) hold. Thus, the first equality of (iii) is proved. 
From (ii), we know that there exists an orthonormal matrix $U$ such that

$$
Z_{p}=\left[Y_{2 p-1}, Y_{2 p}\right] U
$$

This implies that

$$
M Z_{p}=\left[M Y_{2 p-1}, M Y_{2 p}\right] U
$$

On the other hand, by the definitions of $Y_{2 p-1}$ and $\widetilde{Z}_{p-1}$, we have

$$
\operatorname{span}\left\{M Y_{2 p-1}\right\} \subset \operatorname{span}\left\{\widetilde{Z}_{p-1}\right\}
$$

From (3.13) and (3.14),

$$
\left(Z_{p} Z_{p}^{T}\right) M Z_{p}=\left[0, Z_{p} Z_{p}^{T} M Y_{2 p}\right] U .
$$

Hence, we get

$$
\operatorname{span}\left\{\left(Z_{p} Z_{p}^{T}\right) M Z_{p}\right\}=\operatorname{span}\left\{\left(Z_{p} Z_{p}^{T}\right) M Y_{2 p}\right\} .
$$

Furthermore, from (3.5) and (3.12), we have

$$
\operatorname{span}\left\{\left(Z_{p} Z_{p}^{T}\right) M Y_{2 p}\right\}=\operatorname{span}\left\{\left(Z_{p} Z_{p}^{T}\right) M Z_{p}\right\} \subset \operatorname{span}\left\{Y_{2 p-1}\right\} .
$$

This implies

$$
\operatorname{dim}\left(\operatorname{span}\left\{\left(Z_{p} Z_{p}^{T}\right) M Y_{2 p}\right\}\right) \leq \operatorname{dim}\left(\operatorname{span}\left\{Y_{2 p-1}\right\}\right) .
$$

Suppose the inequality of (3.15) holds. Then, from (3.10), we conclude that there exists a vector $\xi \neq 0$ such that

$$
\left(Z_{p} Z_{p}^{T}\right) M Y_{2 p} \xi=0
$$

This implies $M Y_{2 p} \xi \in \operatorname{span}\left\{\widetilde{Z}_{p-1}\right\}$. By the same argument as (3.11) we get the contradiction. Therefore, the second equality of (iii) is proved.

(iv) From (ii) and (iii) immediately follows (iv).

Remark. From statements (iii) and (iv) of Theorem 3.2, we see that the matrices $Y_{2 p-1}$ and $Y_{2 p}$ can be replaced by an orthonormal basis of $\operatorname{span}\left\{\left(Z_{p} Z_{p}^{T}\right) M Z_{p}\right\}$ and $\operatorname{span}\left\{\left(I-W_{2 p-1} W_{2 p-1}^{T}\right) Z_{p}\right\}$, respectively. In the following, we develop an algorithm for computing $Y_{2 p-1}$ and $Y_{2 p}$ by using the orthonormal bases $Z_{p}$.

Algorithm 3.3. This algorithm computes $Y_{2 p-1}$ and $Y_{2 p}$ by using the orthonormal basis $Z_{p}, p=1, \ldots, m_{k}$, obtained by Algorithm 3.1.

Step 1. Compute an orthonormal basis $Q_{1}^{(0)}$ of $M Z_{1}^{(0)}$ and set

$$
Y_{1}^{(0)}=Q_{1}^{(0)} .
$$

Step 2. Compute the SVD of $\left(Q_{1}^{(0)}\right)^{T} Z_{1}^{(0)}$ such that

$$
\left(U_{1}^{(0)}\right)^{T}\left(\left(Q_{1}^{(0)}\right)^{T} Z_{1}^{(0)}\right) V_{1}^{(0)}=\left[\begin{array}{ll}
\Sigma_{1}^{(0)} & 0
\end{array}\right]
$$


where $U_{1}^{(0)}, V_{1}^{(0)}$ are two unitary matrices and

$$
\Sigma_{1}^{(0)}=\left[\begin{array}{lll}
\sigma_{1} & & \\
& \ddots & \\
& & \sigma_{k}
\end{array}\right] .
$$

Set

$$
Y_{2}^{(0)}=Z_{1}^{(0)} V_{1}^{(0)}\left[\begin{array}{c}
0 \\
I_{k}
\end{array}\right] .
$$

Set $p=2$.

\section{Step 3. Repeat:}

If $p>\frac{m_{k}}{2}+1$, then stop.

Determine $j \in\{0,1, \ldots, k-1\}$ such that $m_{j}<p \leq m_{j+1}$.

3.1 Compute an orthonormal basis $Q_{p}^{(j)}$ of $M Z_{p}^{(j)}$.

3.2 Compute the $S V D$ of $\left(Z_{p}^{(j)}\right)^{T} Q_{p}^{(j)}$ such that

$$
\left(U_{2 p-1}^{(j)}\right)^{T}\left[\left(Z_{p}^{(j)}\right)^{T} Q_{p}^{(j)}\right] V_{2 p-1}^{(j)}=\Sigma_{2 p-1}^{(j)},
$$

where $U_{2 p-1}^{(j)}, V_{2 p-1}^{(j)}$ are two unitary matrices and

$$
\Sigma_{2 p-1}^{(j)}=\left[\begin{array}{cccc}
\sigma_{1}^{(2 p-1)} & & & \\
& \ddots & & \\
& & \sigma_{k-j}^{(2 p-1)} & \\
& & & O_{k-j}
\end{array}\right]
$$

with $\sigma_{1}^{(2 p-1)} \geq \cdots \geq \sigma_{k-j}^{(2 p-1)}>0$.

Set

$$
Y_{2 p-1}^{(j)}=Z_{p}^{(j)} U_{p}^{(j)}\left[\begin{array}{c}
I_{k-j} \\
0
\end{array}\right]
$$

3.3 Compute the SVD of $\left(Y_{2 p-1}^{(j)}\right)^{T} Z_{p}^{(j)}$ such that

$$
\left(U_{2 p}^{(j)^{T}}\right)\left[\left(Y_{2 p-1}^{(j)}\right)^{T} Z_{p}^{(j)}\right] V_{2 p}^{(j)}=\left[\Sigma_{2 p}^{(j)} \mid O\right]
$$

where $U_{2 p}^{(j)}, V_{2 p}^{(j)}$ are two unitary matrices and

$$
\Sigma_{2 p}^{(j)}=\left[\begin{array}{ccc}
\sigma_{1}^{(2 p)} & & \\
& \ddots & \\
& & \sigma_{k-j}^{(2 p)}
\end{array}\right]
$$

Set

$$
Y_{2 p}^{(j)}=Z_{p}^{(j)} V_{2 p}^{(j)}\left[\begin{array}{c}
0 \\
I_{k-j}
\end{array}\right]
$$




\subsection{Update $p:=p+1$ and go to Repeat.}

This algorithm needs about $O\left(n^{2}\right)$ flops.

Denote $\Upsilon_{s}$ as an orthonormal basis of the $M$-stable isotropic subspace corresponding to the first half of Jordan blocks of zero eigenvalues. We now define a sequence of orthonormal bases $\left\{\widetilde{\Upsilon}_{p}\right\}_{p=1}^{m_{k}}$ which is closely related to the matrix $\Upsilon_{s}$.

DEFINITION 3.4. Let $\widetilde{\Upsilon}_{p}$ for $p=1, \ldots, m_{k}$ be a maximal orthonormal basis satisfying the following:

(i) $\operatorname{span}\left\{\widetilde{\Upsilon}_{p}\right\} \subset \mathcal{N}\left(M^{p}\right)$ (null space of $\left.M^{p}\right)$.

(ii) $x^{T} J y=0$ for any $x, y \in \operatorname{span}\left\{\widetilde{\Upsilon}_{p}\right\}$.

(iii) $\operatorname{span}\left\{\widetilde{\Upsilon}_{p-1}\right\} \subset \operatorname{span}\left\{\widetilde{\Upsilon}_{p}\right\} .\left(\right.$ Here,,$\widetilde{\Upsilon}_{0} \equiv 0$.)

(iv) If there is a subspace $\mathcal{V} \subset \mathbf{R}^{2 n}$ satisfying statements (i), (ii), and (iii), then $\mathcal{V} \subset \operatorname{span}\left\{\widetilde{\Upsilon}_{p}\right\}$.

THEOREM 3.5. The following properties for the sequence $\left\{\widetilde{\Upsilon}_{p}\right\}_{p=1}^{m_{k}}$ defined above are true:

(i) $\operatorname{span}\left\{\widetilde{\Upsilon}_{p}\right\}$ is unique for $p \in\left\{1, \ldots, m_{k}\right\}$.

(ii) $\operatorname{span}\left\{\widetilde{\Upsilon}_{m_{k}}\right\}=\operatorname{span}\left\{\Upsilon_{s}\right\}$.

Proof. (i) From Theorem 2.1 and assumption (A1), we can assume that $M$ has the form (2.2). Since $\operatorname{span}\left\{\widetilde{\Upsilon}_{p}\right\} \subset \mathcal{N}\left(M^{p}\right)$ for $p=1, \ldots, m_{k}$, for convenience, we assume without loss of generality (w.l.o.g.) that $M$ has only zero eigenvalues and discuss two typical cases of $M$ in the following.

Case 1. Let $k=2, m_{1}<m_{2}$, and

$$
M=\left[\begin{array}{cccc}
J^{\left(m_{1}\right)}(0) & & \Lambda^{\left(m_{1}\right)}(0) & \\
& J^{\left(m_{2}\right)}(0) & & \Lambda^{\left(m_{2}\right)}(0) \\
& & -J^{\left(m_{1}\right)}(0)^{T} & -J^{\left(m_{2}\right)}(0)^{T}
\end{array}\right]
$$

with $\Lambda^{\left(m_{1}\right)}(0)\left(m_{1}, m_{1}\right)=\Lambda^{\left(m_{2}\right)}(0)\left(m_{2}, m_{2}\right)=1$.

For $p \leq m_{1}$, we have

$$
\mathcal{N}\left(M^{p}\right)=\operatorname{span}\left\{e_{1}, \ldots, e_{p}, e_{m_{1}+1}, \ldots, e_{m_{1}+p}\right\} .
$$

Since $p<m_{1}+p \leq m_{1}+m_{2}$ (= the half of dimension of $M$ ) for any $x, y \in \mathcal{N}\left(M^{p}\right)$ we have $x^{T} J y=0$. From the definition of $\widetilde{\Upsilon}_{p}$ it follows that

$$
\operatorname{span}\left\{\widetilde{\Upsilon}_{p}\right\}=\mathcal{N}\left(M^{p}\right) .
$$

In addition, $\mathcal{N}\left(M^{p}\right)$ is unique. Thus, $\operatorname{span}\left\{\widetilde{\Upsilon}_{p}\right\}$ is unique for $p \leq m_{1}$.

For $m_{1}+1 \leq p \leq m_{2}$, we have

$\mathcal{N}\left(M^{p}\right)=\operatorname{span}\left\{e_{1}, \ldots, e_{m_{1}}, e_{\left(m_{1}+m_{2}\right)+m_{1}}, \ldots, e_{\left(m_{1}+m_{2}\right)+m_{1}-p+1}, e_{m_{1}+1}, \ldots, e_{m_{1}+p}\right\}$.

Let $\mathcal{U} \equiv \operatorname{span}\left\{e_{1}, \ldots, e_{m_{1}}, e_{m_{1}+1}, \ldots, e_{m_{1}+p}\right\}$. Obviously, $\mathcal{U} \subset \mathcal{N}\left(M^{p}\right)$. Since $m_{1}+$ $p<m_{1}+m_{2}$ for any $x, y \in \mathcal{U}$ we have $x^{T} J y=0$. Hence,

$$
\mathcal{U} \subset \operatorname{span}\left\{\widetilde{\Upsilon}_{p}\right\}
$$

If $\mathcal{U} \neq \operatorname{span}\left\{\widetilde{\Upsilon}_{p}\right\}$, then there exists a nonzero vector $v \in \operatorname{span}\left\{e_{\left(m_{1}+m_{2}\right)+m_{1}}, \ldots\right.$, $\left.e_{\left(m_{1}+m_{2}\right)+m_{1}-p+1}\right\}$ such that $v \in \operatorname{span}\left\{\widetilde{\Upsilon}_{p}\right\}$ and $v \notin \mathcal{U}$. But, for this $v$, there exists an associated nonzero vector $u \in \mathcal{U}$ such that

$$
u^{T} J v \neq 0 .
$$


This contradicts the definition of $\operatorname{span}\left\{\widetilde{\Upsilon}_{p}\right\}$. Hence $\mathcal{U}=\operatorname{span}\left\{\widetilde{\Upsilon}_{p}\right\}$. Since $\mathcal{U}$ is unique, the proof follows.

Case 2. Let $k=3,2 m_{1}<m_{2}$, and

$$
M=\left[\begin{array}{cc|cc}
J^{\left(2 m_{1}\right)}(0) & & 0 & \\
& J^{\left(m_{2}\right)}(0) & & \Lambda^{\left(m_{2}\right)}(0) \\
& & -J^{\left(2 m_{1}\right)}(0)^{T} & \\
& & & -J^{\left(m_{2}\right)}(0)^{T}
\end{array}\right]
$$

with $\Lambda^{\left(m_{2}\right)}(0)\left(m_{2}, m_{2}\right)=1$. The proof of this case is similar to that for Case 1 . We omit it here.

(ii) By the definition of $\operatorname{span}\left\{\widetilde{\Upsilon}_{p}\right\}$ and (i), (ii) follows immediately.

Remark. If we ignore the monotone property of $\operatorname{span}\left\{\widetilde{\Upsilon}_{p}\right\}$, i.e., condition (iii) of Definition 3.4, then the uniqueness of $\operatorname{span}\left\{\widetilde{\Upsilon}_{p}\right\}$ does not hold. For example, let $2 m_{1}=2,2 m_{2}=4$, and

$$
M=\left[\begin{array}{rrrrrr}
0 & 0 & 0 & 1 & 0 & 0 \\
0 & 0 & 1 & 0 & 0 & 0 \\
0 & 0 & 0 & 0 & 0 & 1 \\
0 & 0 & 0 & 0 & 0 & 0 \\
0 & 0 & 0 & 0 & 0 & 0 \\
0 & 0 & 0 & 0 & -1 & 0
\end{array}\right]
$$

then for $p=2$ there exist two different maximal isotropic orthonormal bases $\left\{e_{1}^{(6)}, e_{2}^{(6)}\right.$, $\left.e_{3}^{(6)}\right\}$ and $\left\{e_{2}^{(6)}, e_{3}^{(6)}, e_{4}^{(6)}\right\}$. But the latter does not form a subspace of $\operatorname{span}\left\{\Upsilon_{s}\right\}$. Hence, we must determine $\Upsilon_{s}$ by using a monotone process.

We now develop an algorithm to determine the maximal isotropic subbasis $\Upsilon_{s}$ by using the computed $\widetilde{Y}_{m_{k}} \equiv\left[Y_{1}, \ldots, Y_{m_{k}}\right]$ and Theorem 3.5.

ALGORITHM 3.6. This algorithm computes $\Upsilon_{s}$ by using orthonormal basis $\widetilde{Y}_{m_{k}}$ obtained by Algorithm 3.3 .

Step 1. Let $\widehat{\Upsilon}=\left[Y_{1}^{(0)}, \ldots, Y_{m_{1}}^{(0)}\right]$ and $\widehat{p}=m_{1}+1$.

Step 2. Repeat:

Determine $j \in\{1, \ldots, k\}$ is a maximal integer such that $m_{j}<\widehat{p}$.

If $j=k$, set $\Upsilon_{s}=\widehat{\Upsilon}$ and stop.

For $p=m_{j}+1, \ldots, m_{j+1}$ :

2.1 Find $i \geq 0$ such that $2 m_{i}<p \leq 2 m_{i+1}$.

If $p=m_{1}+1$, then $\widehat{Y}_{m_{1}+1}^{(0)}=Y_{m_{1}+1}^{(0)}$,

else $\widehat{Y}_{p}^{(i)}=\left[Y_{m_{1}+1}^{(0)}, \ldots, Y_{p}^{(i)}\right]$.

Let $\# 1=$ the number of columns of $\widehat{\Upsilon}$.

Let $\# 2$ = the number of columns of $\widehat{Y}_{p}^{(i)}$.

2.2 Compute the $S V D$ of $\widehat{\Upsilon}^{T} J \widehat{Y}_{p}^{(i)}$ such that

$$
\left(U_{p}^{(j)}\right)^{T}\left[\widehat{\Upsilon}^{T} J \widehat{Y}_{p}^{(i)}\right] V_{p}^{(j)}=\left[\Sigma_{p}^{(j, i)} \mid O\right],
$$

where

$$
\Sigma_{p}^{(j, i)}=\left[\begin{array}{ccc}
\sigma_{1}^{(j, i)} & & \\
& \ddots & \\
& & \sigma_{\# 1}^{(j, i)}
\end{array}\right]
$$


Let $\# 3=\max \left\{q \mid \sigma_{q}^{(j, i)}>0\right.$ for $\left.q=1, \ldots, \# 1\right\}$.

2.3 Update $\widehat{\Upsilon}=\left[Y_{1}^{(0)}, \ldots, Y_{m_{1}}^{(0)}, \widehat{Y}_{p}^{(i)} V_{p}^{(j)}\left[\begin{array}{c}0 \\ I_{\# 2-\# 3}\end{array}\right]\right]$.

Endfor.

Step 3. Update $\widehat{p}=p+1$ and go to Repeat.

Comment: (i) In substep 2.1, it is easily seen that

$$
\# 1=\sum_{\ell=0}^{j-1}\left(m_{\ell+1}-m_{\ell}\right)(k-\ell)+\left(p-m_{j}\right)(k-j)
$$

and

$$
\# 2=2 \sum_{\ell=0}^{i-1}\left(m_{\ell+1}-m_{\ell}\right)(k-\ell)+\left(p-2 m_{i}\right)(k-i)-m_{1} k .
$$

(ii) This algorithm needs about $O\left(n^{2}\right)$ flops.

After the $M$-stable isotropic subspace $\operatorname{span}\left\{\Upsilon_{s}\right\}$ is found, we can deflate it by using symplectic orthogonal transformations to get a reduced Hamiltonian matrix $\widehat{M}$ (say!) having no purely imaginary eigenvalue. Then we compute the maximal stable isotropic subspace of $\widehat{M}$ by exploiting [2, 23, 29]. Combining these two computed isotropic subspaces, we obtain the desired $M$-stable Lagrangian subspace $\mathcal{Y}_{\mathcal{L}}$.

4. Computing the stable Lagrangian subspace of a symplectic pencil having unimodular eigenvalues. Let $N-\lambda L$ be a symplectic pencil as in (1.2). Assume (A2) holds; i.e., the partial multiplicities of unimodular eigenvalues of $N-\lambda L$ are all even. In this section, we shall develop an algorithm to compute the $(N, L)$-stable isotropic subspace $\mathcal{W}$ corresponding to the first half Jordan blocks of all unimodular eigenvalues and get a reduced symplectic pencil having no unimodular eigenvalue. Combining $\mathcal{W}$ with the maximal isotropic subspace corresponding to the strictly stable eigenvalues of $N-\lambda L$, we obtain the desired $(N, L)$-stable Lagrangian subspace $\mathcal{W}_{\mathcal{L}}$.

The main idea of our algorithm to determine $\mathcal{W}$ is that by using $S+S^{-1}$ transformation [18] we first compute a Jordan basis of $\Gamma-\lambda \Delta$ as in (1.7) corresponding to eigenvalues with magnitudes between -2 and 2 and a Jordan basis corresponding to unimodular eigenvalues of $N-\lambda L$ and then use it to determine an isotropic basis $\Upsilon$ of $\mathcal{W}$.

We recall from (1.7) that

$$
\Gamma-\lambda \Delta \equiv\left[\left(N J L^{T}+L J N^{T}\right)-\lambda L J L^{T}\right] J^{T} .
$$

Now we want to show the relation between Jordan bases corresponding to the unimodular eigenvalue $\mu$ of $N-\lambda L$ and the eigenvalue $\mu+\mu^{-1}$ of $\Gamma-\lambda \Delta$, respectively. For the pencil $N-\lambda L$, we can use the method of [20, p. 120] to deflate its zero and infinity eigenvalues simultaneously and get a reduced symplectic pencil having no zero or infinity eigenvalues. Hence, we can assume w.l.o.g. that both $N$ and $L$ are nonsingular in the following.

THEOREM 4.1. Let $N-\lambda L$ be a symplectic pencil having unimodular eigenvalues $\mu \in\left\{ \pm 1, e^{ \pm i \theta},(\theta \neq 0)\right\}$. Let

$$
J^{\left(2 m_{1}\right)}(\mu), \ldots, J^{\left(2 m_{k}\right)}(\mu)
$$

be the corresponding Jordan blocks with even sizes. Then 
(i) for $\mu= \pm 1$ the corresponding eigenvalue 2 or -2 of $\Gamma-\lambda \Delta$ has Jordan blocks

$$
J^{\left(m_{1}\right)}( \pm 2), J^{\left(m_{1}\right)}( \pm 2), \ldots, J^{\left(m_{k}\right)}( \pm 2), J^{\left(m_{k}\right)}( \pm 2),
$$

(ii) for $\mu=e^{ \pm i \theta}$ the corresponding eigenvalue $e^{i \theta}+e^{-i \theta}$ of $\Gamma-\lambda \Delta$ has Jordan blocks

$$
J^{\left(2 m_{1}\right)}\left(e^{i \theta}+e^{-i \theta}\right), \ldots, J^{\left(2 m_{k}\right)}\left(e^{i \theta}+e^{-i \theta}\right)
$$

with the same sizes as (4.1).

Proof. To prove this theorem, we consider the following simple case. The complete proof is a straightforward generalization. Let $Y=\left[y_{1}, \ldots, y_{2 m_{1}}\right]$ be a Jordan basis of $J^{\left(2 m_{1}\right)}(\mu)$ satisfying

$$
N Y=L Y J^{\left(2 m_{1}\right)}(\mu)
$$

Write $Y=J L^{T} J^{T} Z$ with $Z=\left[z_{1}, \ldots, z_{2 m_{1}}\right]$. Substituting $Y$ into (4.2), we have

$$
N J L^{T} J^{T} Z=L J L^{T} J^{T} Z J^{\left(2 m_{1}\right)}(\mu) .
$$

Since $N J N^{T}=L J L^{T}$ and $N$ and $J^{\left(2 m_{1}\right)}(\mu)$ are invertible, from (4.3) we get

$$
L J N^{T} J^{T} Z=L J L^{T} J^{T} Z J^{\left(2 m_{1}\right)}(\mu)^{-1} .
$$

Combining (4.3) and (4.4), we get

$$
\left(N J L^{T} J^{T}+L J N^{T} J^{T}\right) Z=L J L^{T} J^{T} Z\left(J^{\left(2 m_{1}\right)}(\mu)+J^{\left(2 m_{1}\right)}(\mu)^{-1}\right) .
$$

If $\mu= \pm 1$, then it is easily seen that

$$
\begin{aligned}
J^{\left(2 m_{1}\right)}( \pm 1)+J^{\left(2 m_{1}\right)}( \pm 1)^{-1} \stackrel{s}{\sim}\left[\begin{array}{ccccc} 
\pm 2 & 0 & \pm 1 & & 0 \\
& & & \ddots & \\
& & \ddots & \ddots & \pm 1 \\
& & & 0 \\
0 & & & \pm 2
\end{array}\right] \\
\stackrel{s}{\sim}\left[\begin{array}{ccc}
J^{\left(m_{1}\right)}( \pm 2) & 0 \\
0 & & J^{\left(m_{1}\right)}( \pm 2)
\end{array}\right] .
\end{aligned}
$$

Here the symbol $\stackrel{s}{\sim}$ denotes "similar." Thus, statement (i) is proved.

If $\mu=e^{ \pm i \theta}$, then it is easily seen that

$$
J^{\left(2 m_{1}\right)}\left(e^{ \pm i \theta}\right)+J^{\left(2 m_{1}\right)}\left(e^{ \pm i \theta}\right)^{-1} \stackrel{s}{\sim}\left[\begin{array}{cccc}
e^{i \theta}+e^{-i \theta} & 1 & & \\
& \ddots & \ddots & \\
& & \ddots & 1 \\
& & & e^{i \theta}+e^{-i \theta}
\end{array}\right] .
$$

Hence, statement (ii) follows.

As in section 3, we can also give the relation between orthonormal Jordan bases corresponding to eigenvalues 2 and 1 of $\Gamma-\mu \Delta$ and $N-\lambda L$, respectively. Here we use the same notation as in section 3 . 
Let $\widetilde{Z}_{q}=\left[Z_{1}, \ldots, Z_{q}\right]$ and $\widetilde{Y}_{p}=\left[Y_{1}, \ldots, Y_{p}\right]$ for $q=1, \ldots, m_{k}$ and $p=1, \ldots, 2 m_{k}$ be the orthonormal Jordan bases corresponding to 2 and 1 of $\Gamma-\mu \Delta$ and $N-$ $\lambda L$, respectively, where $Z_{q}$ and $Y_{p}$ are orthonormal Jordan bases of degree $q$ and $p$, respectively. We say that $Z_{q}$ is of degree $q$ if it holds

$$
(\Gamma-2 \Delta) v \in \operatorname{span}\left\{\Delta \widetilde{Z}_{q-1}\right\}, \quad(\Gamma-2 \Delta) v \notin \operatorname{span}\left\{\Delta \widetilde{Z}_{q-2}\right\} \quad(\text { for } q \geq 2)
$$

for all $v \in \operatorname{span}\left\{Z_{q}\right\}$ and that $Y_{p}$ is of degree $p$ if it holds

$$
(N-L) v \in \operatorname{span}\left\{L \widetilde{Y}_{p-1}\right\}, \quad(N-L) v \notin \operatorname{span}\left\{L \widetilde{Y}_{p-2}\right\} \quad(\text { for } p \geq 2)
$$

for all $v \in \operatorname{span}\left\{Y_{p}\right\}$. Here we set $\widetilde{Z}_{0}=0$ and $\widetilde{Y}_{0}=0$.

Let $\Theta_{q}$ be an orthonormal basis of $J L^{T} J^{T} Z_{q}$ and $\widetilde{\Theta}_{q}=\left[\Theta_{1}, \ldots, \Theta_{q}\right]$ for $q=$ $1, \ldots, m_{k}$.

TheOREM 4.2. For $p=1, \ldots, m_{k}$, we have

(i) $\operatorname{span}\left\{\widetilde{\Theta}_{p}\right\}=\operatorname{span}\left\{\widetilde{Y}_{2 p}\right\}$.

(ii) $\operatorname{span}\left\{\Theta_{p}\right\}=\operatorname{span}\left\{Y_{2 p-1}\right\} \oplus \operatorname{span}\left\{Y_{2 p}\right\}$,

(iii) $\operatorname{span}\left\{Y_{2 p-1}\right\}=\operatorname{span}\left\{\left(I-\widetilde{\Theta}_{p-1} \widetilde{\Theta}_{p-1}^{T}\right)\left(L^{-1} N-I\right) \Theta_{p}\right\}$

$$
=\operatorname{span}\left\{\left(\Theta_{p} \Theta_{p}^{T}\right)\left(L^{-1} N-I\right) \Theta_{p}\right\}
$$

(iv) if $W_{2 p-1}$ is an orthonormal basis of $\operatorname{span}\left\{\left(I-\widetilde{\Theta}_{p-1} \widetilde{\Theta}_{p-1}^{T}\right)\left(L^{-1} N-I\right) \Theta_{p}\right\}$, then $\operatorname{span}\left\{Y_{2 p}\right\}=\operatorname{span}\left\{\left(I-W_{2 p-1} W_{2 p-1}^{T}\right) \Theta_{p}\right\}$.

Proof. (i) Let $p=1$. For $u \in \operatorname{span}\left\{\widetilde{\Theta}_{1}\right\}$ there is a vector $v \in \operatorname{span}\left\{\widetilde{Z}_{1}\right\}$ such that $u=J L^{T} J^{T} v$. Then we have $(\Gamma-2 \Delta) v=0$. Since

$$
N L^{-1}(\Gamma-2 \Delta)=(N-L) L^{-1}(N-L) J L^{T} J^{T} \quad(\text { from }(1.7)),
$$

we have

$$
0=N L^{-1}(\Gamma-2 \Delta) v=(N-L) L^{-1}(N-L) J L^{T} J^{T} v .
$$

Hence, $u \in \operatorname{span}\left\{\widetilde{Y}_{2}\right\}$ and $\operatorname{span}\left\{\widetilde{\Theta}_{1}\right\} \subset \operatorname{span}\left\{\widetilde{Y}_{2}\right\}$.

Conversely, if $u \in \operatorname{span}\left\{\widetilde{Y}_{2}\right\}$, then

$$
(N-L) L^{-1}(N-L) u=0 .
$$

By (4.5) and (4.6), we have

$$
N L^{-1}(\Gamma-2 \Delta)\left(J L^{T} J^{T}\right)^{-1} u=0 .
$$

Let $v=\left(J L^{T} J^{T}\right)^{-1} u$. Since $N$ and $L$ are nonsingular, we have $(\Gamma-2 \Delta) v=0$. Thus, $v \in \operatorname{span}\left\{\widetilde{Z}_{1}\right\}$. Statement (i) holds for $p=1$.

Assume that statement (i) holds for $\widehat{p}=p-1<m_{k}$. For $u \in \operatorname{span}\left\{\widetilde{\Theta}_{p}\right\}$ there is a vector $v \in \operatorname{span}\left\{\widetilde{Z}_{p}\right\}$ such that $u=J L^{T} J^{T} v$. By (4.5) and the definition of $\widetilde{Z}_{p}$, we have

$$
\begin{aligned}
(N-L) L^{-1}(N-L)\left(J L^{T} J^{T}\right) v & =N L^{-1}(\Gamma-2 \Delta) v \\
& =N L^{-1} \Delta\left(\widetilde{Z}_{p-1} \widetilde{w}_{p-1}\right) \\
& =N\left(J L^{T} J^{T} \widetilde{Z}_{p-1} \widetilde{w}_{p-1}\right)
\end{aligned}
$$

for some nonzero vector $\widetilde{w}_{p-1}$. Since (i) holds for $\widehat{p}=p-1$, there is a nonzero vector $\widehat{w}_{2(p-1)}$ such that

$$
\widetilde{Y}_{2(p-1)} \widehat{w}_{2(p-1)}=J L^{T} J^{T} \widetilde{Z}_{p-1} \widetilde{w}_{p-1}
$$


This implies

$$
\begin{aligned}
(N-L) L^{-1}(N-L)\left(J L^{T} J^{T}\right) v & =N \widetilde{Y}_{2(p-1)} \widehat{w}_{2(p-1)} \\
& =(N-L) \widetilde{Y}_{2(p-1)} \widehat{w}_{2(p-1)}+L \widetilde{Y}_{2(p-1)} \widehat{w}_{2(p-1)} \\
& \in \operatorname{span}\left\{L \widetilde{Y}_{2(p-1)}\right\} .
\end{aligned}
$$

Hence, $u \in \operatorname{span}\left\{\widetilde{Y}_{2 p}\right\}$.

Conversely, if $u \in \operatorname{span}\left\{\widetilde{Y}_{2 p}\right\}$, from the proof of (i) of Theorem 4.1, we know that there is a nonzero vector $v$ with $u=J L^{T} J^{T} v$ such that $v \in \operatorname{span}\left\{\widetilde{Z}_{p}\right\}$. Hence, by induction, statement (i) follows.

(ii), (iii), (iv) From (i) we have that

$$
\operatorname{span}\left\{\left(L^{-1} N-I\right) \Theta_{p}\right\} \subset \operatorname{span}\left\{\widetilde{Y}_{2 p-1}\right\} .
$$

Using (4.7) and a similar argument as in Theorem 3.2, we obtain (ii), (iii), and (iv) immediately.

According to Theorems 4.1, 4.2, and 3.5, we can also develop a structure-preserving algorithm to compute the $(N, L)$-stable Lagrangian subspace $\mathcal{W}_{\mathcal{L}}$. The algorithm is similar to Algorithms 3.1, 3.3, and 3.6. We omit the detail descriptions while the statements are the same.

AlgORITHM 4.3. This algorithm computes the desired $(N, L)$-stable isotropic basis $\Upsilon_{s}$. Suppose that the only unimodular eigenvalue of $N-\lambda L$ is one.

Step 1: Reduce the skew-Hamiltonian pencil $\Gamma-\lambda \Delta \equiv\left[\left(N J L^{T}+L J N^{T}\right)-\right.$ $\left.\lambda L J L^{T}\right] J^{T}$ to a skew-Hamiltonian quasi-upper upper triangular pencil by using the stable algorithm proposed by [22]; i.e., find orthogonal matrices $U$ and $Q$ such that

$$
U^{T} \Gamma Q=\left[\begin{array}{cc}
\Gamma_{1} & H_{1} \\
O & \Gamma_{1}^{T}
\end{array}\right] \equiv H
$$

and

$$
U^{T} \Delta Q=\left[\begin{array}{cc}
\Delta_{1} & E_{1} \\
O & \Delta_{1}^{T}
\end{array}\right] \equiv E,
$$

where $\Gamma_{1}$ is quasi-upper triangular, $\Delta_{1}$ is upper triangular, and $H_{1}, E_{1}$ are skew symmetric.

Set

$$
\begin{aligned}
& H:=\left[\begin{array}{cc}
I & O \\
O & \hat{I}
\end{array}\right] H\left[\begin{array}{cc}
I & O \\
O & \hat{I}
\end{array}\right] \quad \text { (quasi-upper triangular), } \\
& E:=\left[\begin{array}{cc}
I & O \\
O & \hat{I}
\end{array}\right] E\left[\begin{array}{cc}
I & O \\
O & \hat{I}
\end{array}\right] \quad \text { (upper triangular), } \\
& Q:=Q\left[\begin{array}{cc}
I & O \\
O & \hat{I}
\end{array}\right] \text {, where } \hat{I}=\left[\begin{array}{ccc}
0 & & 1 \\
& . & \\
& \cdot & \\
1 & & 0
\end{array}\right] \text {. }
\end{aligned}
$$

Let $j=0, q=1$. 
Step 2: Compute $Z_{q}^{(j)}$ for $q=1, \ldots, m_{k}$, by performing the same statements in Step 3 of Algorithm 3.1 but replacing $H$ by $H-2 E$. Compute an orthonormal basis $\Theta_{q}^{(j)}$ of $J L^{T} J^{T} Z_{q}^{(j)}$ for $q=1, \ldots, m_{k}$.

Comment: Here $Z_{q}^{(j)}$ is an orthonormal Jordan basis of degree $q$ corresponding to eigenvalue 2 of $\Gamma-\lambda \Delta$.

Step 3: Perform the same statements as in Algorithm 3.3 but replace $M Z_{p}^{(j)}$ by $\left(L^{-1} N-I\right) \Theta_{p}^{(j)}$.

Comment: This step computes an orthonormal Jordan basis $\left\{Y_{p}^{(j)}\right\}_{p=1}^{m_{k}}$ of $N-\lambda L$ corresponding to the unimodular eigenvalue 1 .

Step 4: Perform the same statements as in Algorithm 3.6 to compute the desired $(N, L)$-stable isotropic basis $\Upsilon_{s}$.

Remark. If $N-\lambda L$ has unimodular eigenvalues -1 or $e^{ \pm i \theta}$, then we replace $H-2 E$ in Step 2 by $H+2 E$ or $H-\eta E$ with $\eta=e^{i \theta}+e^{-i \theta}$ and perform the same process.

According to Algorithm 4.3, we can find the desired $(N, L)$-stable isotropic basis

$$
\Upsilon_{s} \equiv\left[\Upsilon_{11}^{(0)^{T}}, \Upsilon_{21}^{(0)^{T}}, \Upsilon_{31}^{(0)^{T}}, \Upsilon_{41}^{(0)^{T}}\right]^{T}
$$

with $\Upsilon_{11}^{(0)}, \Upsilon_{31}^{(0)} \in \mathbf{R}_{2}^{n_{0}} \times \frac{n_{0}}{2}$ of $N-\lambda L$. Here, $n_{0}$ is the number of unimodular eigenvalues. We now give an algorithm to determine a symplectic matrix $Q$ and a nonsingular $U$ such that

$$
U(N-\lambda L) Q=\left[\begin{array}{cccc}
N_{11} & N_{12} & 0 & 0 \\
0 & N_{22} & 0 & 0 \\
0 & 0 & I & 0 \\
0 & N_{42} & 0 & I
\end{array}\right]-\lambda\left[\begin{array}{cccc}
I & 0 & L_{13} & L_{14} \\
0 & I & L_{23} & L_{24} \\
0 & 0 & L_{33} & 0 \\
0 & 0 & L_{43} & L_{44}
\end{array}\right],
$$

where the reduced symplectic pencil $\left[\begin{array}{cc}N_{22} & 0 \\ N_{42} & I\end{array}\right]-\lambda\left[\begin{array}{ll}I & L_{24} \\ 0 & L_{44}\end{array}\right]$ has no unimodular eigenvalue. Here $L_{44}=N_{22}^{T}, N_{42}=N_{42}^{T}$, and $L_{24}=L_{24}^{T}$.

ALGORITHM 4.4. This algorithm is to determine a symplectic matrix $Q$ and an invertible matrix $U$ such that (4.8) holds.

Step 1: Find a symplectic Householder matrix $Q_{1}$ such that

$$
Q_{1}^{T} \Upsilon_{s}=\left[\begin{array}{c}
\Upsilon_{11}^{(1)} \\
\Upsilon_{21}^{(1)} \\
\Upsilon_{31}^{(1)} \\
0
\end{array}\right]
$$

Set

$$
N:=Q_{1}^{T} N Q_{1}, \quad L:=Q_{1}^{T} L Q_{1}, \quad U:=Q_{1}^{T}, \quad Q:=Q_{1} .
$$

Step 2: If $\Upsilon_{11}^{(1)}$ is singular or ill conditioned, then Return.

Else compute a Gaussian symplectic matrix $Q_{2}^{-1} \equiv\left[\begin{array}{ll}I & 0 \\ \Omega & I\end{array}\right]$, with $\Omega \equiv-\Upsilon_{31}^{(1)} \Upsilon_{11}^{(1)}{ }^{-1}$, so that

$$
Q_{2}^{-1}\left[\begin{array}{c}
\Upsilon_{11}^{(1)} \\
\Upsilon_{21}^{(1)} \\
\Upsilon_{31}^{(1)} \\
0
\end{array}\right]=\left[\begin{array}{c}
\Upsilon_{11}^{(2)} \\
\Upsilon_{21}^{(2)} \\
0 \\
0
\end{array}\right]
$$


Comment: Since $\Upsilon_{s}$ is isotropic, it follows that $\Omega$ is symmetric. Thus $Q_{2}$ is symplectic.

If $\left(I+L_{12} \Omega\right)$ is singular or ill conditioned, then Return.

Else set

$$
\begin{aligned}
& U_{2}:=\left[\begin{array}{cc}
\left(I+L_{12} \Omega\right)^{-1} & 0 \\
0 & I
\end{array}\right], \quad U_{3}:=\left[\begin{array}{cc}
I & 0 \\
-L_{12} \Omega & I
\end{array}\right], \\
& Q:=Q Q_{2}, \quad U:=U_{3} U_{2} U
\end{aligned}
$$

and form

$$
\begin{aligned}
& N:=U N Q=\left[\begin{array}{ll}
\left(I+L_{12} \Omega\right)^{-1} N_{11} & 0 \\
N_{21}+\Omega-L_{22} \Omega\left(I+L_{12} \Omega\right)^{-1} N_{11} & I
\end{array}\right], \\
& L:=U L Q=\left[\begin{array}{cc}
I & \left(I+L_{12} \Omega\right)^{-1} L_{12} \\
0 & L_{22}-L_{22} \Omega\left(I+L_{12} \Omega\right)^{-1} L_{12}
\end{array}\right] .
\end{aligned}
$$

Comment: Here the matrix $L_{22}-L_{22} \Omega\left(I+L_{12} \Omega\right)^{-1} L_{12}=N_{11}^{T}\left(I+\Omega L_{12}\right)^{-1}$ and $\left(I+L_{12} \Omega\right)^{-1} L_{12}$ is symmetric.

Step 3: Find a symplectic Householder matrix $Q_{3}$ such that

$$
Q_{3}^{T}\left[\begin{array}{c}
\Upsilon_{11}^{(2)} \\
\Upsilon_{21}^{(2)} \\
0 \\
0
\end{array}\right]=\left[\begin{array}{c}
\Upsilon_{11}^{(3)} \\
0 \\
0 \\
0
\end{array}\right]
$$

Set

$$
N:=Q_{3}^{T} N Q_{3}, \quad L:=Q_{3}^{T} L Q_{3}, \quad Q:=Q Q_{3}, \quad U:=Q_{3}^{T} U .
$$

Remark. (i) This algorithm deflates the maximal $(N, L)$-stable isotropic subspace of $N-\lambda L$ corresponding to unimodular eigenvalues and gets a reduced symplectic pencil having no unimodular eigenvalue. Consequently, we can use the structurepreserving algorithm proposed by [19] or [29] to compute the stable invariant subspace of the reduced symplectic pencil. (ii) If the matrix $\left(I+L_{12} \Omega\right)$ or $\Upsilon_{11}^{(1)}$ in Step 2 is not invertible or ill conditioned, then we return the deflation process to $N-\lambda L$. We deflate the isotropic basis $\Upsilon_{s}$ from $N-\lambda L$ directly by using symplectic orthogonal transformations and get a reduced symplectic pencil having no unimodular eigenvalue. Then we apply the algorithm of [29] to find the rest of the stable invariant subspace. Although here only orthogonal symplectic transformations are used, it is numerically difficult to keep symplecticity of $N-\lambda L$ explicitly [7]. Hence, it may be numerically troublesome in this case.

5. Numerical examples. In this section we illustrate the numerical performance of our algorithms for a Hamiltonian matrix $M$. A program based on Algorithms $3.1,3.3$, and 3.6 has been implemented on a SUN 4/470 computer using MATLAB with eps $\approx 10^{-16}$. 
Example 5.1. Let

$$
\begin{aligned}
& A_{0}=\operatorname{diag}\left\{[0], J^{(2)}(0)^{T}, J^{(4)}(0)^{T},-I_{2}\right\}, \\
& H_{0}=\operatorname{diag}\left\{[-1],-\Lambda^{(2)}(0),-\Lambda^{(4)}(0),-I_{2}\right\}, \\
& G_{0}=O_{9 \times 9},
\end{aligned}
$$

where $J^{\left(m_{j}\right)}(0)$ and $\Lambda^{\left(m_{j}\right)}(0)$ are defined in section 1 with $\Lambda^{\left(m_{j}\right)}(0)\left(m_{j}, m_{j}\right)=1$, $j=1,2$. It is easily seen that the corresponding Hamiltonian matrix $M_{0}=\left[\begin{array}{cc}A_{0} & 0 \\ H_{0} & -A_{0}^{T}\end{array}\right]$ has nonzero eigenvalues $-1,-1,1,1$ and the zero eigenvalue with partial multiplicities $\{2,4,8\}$. Now we construct a nontrivial Hamiltonian matrix $M$ by

$$
M=\left[\begin{array}{cc}
I & V_{2} \\
0 & I
\end{array}\right]\left[\begin{array}{cc}
V_{1}^{T} & 0 \\
0 & V_{1}^{-1}
\end{array}\right] M_{0}\left[\begin{array}{cc}
V_{1}^{-T} & 0 \\
0 & V_{1}
\end{array}\right]\left[\begin{array}{cc}
I & -V_{2} \\
0 & I
\end{array}\right],
$$

where

$$
V_{1}=\left[\begin{array}{llll}
1 & 1 & & 0 \\
& \ddots & \ddots & \\
& & & 1 \\
0 & & & 1
\end{array}\right] \text { and } V_{2}=\left[\begin{array}{rrrrrr}
1 & 1 & & & & 0 \\
1 & -1 & 2 & & & \\
& 2 & 1 & \ddots & & \\
& & \ddots & \ddots & & \\
& & & & -1 & 8 \\
0 & & & & 8 & 1
\end{array}\right] \text {. }
$$

The new matrix $M \equiv\left[\begin{array}{cc}A & G \\ H & -A^{T}\end{array}\right]$ has the same Jordan canonical form as $M_{0}$ and has the forms

$$
\begin{aligned}
& A=\left[\begin{array}{rrrrrrrrr}
-1 & -1 & 1 & 0 & 0 & 0 & 0 & 0 & 0 \\
-1 & 3 & -3 & 0 & 0 & 0 & 0 & 0 & 0 \\
-1 & 0 & 1 & 0 & 0 & 0 & 0 & 0 & 0 \\
-1 & 4 & -3 & 0 & 0 & 0 & 0 & 0 & 0 \\
-1 & 1 & -1 & 1 & 0 & -5 & 5 & 0 & 0 \\
-1 & 1 & -1 & 0 & 1 & 7 & -13 & 6 & 0 \\
-1 & 1 & -1 & 0 & 0 & -4 & 11 & -13 & 7 \\
-1 & 1 & -1 & 0 & 0 & 8 & -14 & 16 & -9 \\
-1 & 1 & -1 & 0 & 0 & 1 & 9 & -15 & 6
\end{array}\right] \\
& H=\operatorname{diag}\left\{\left[\begin{array}{rrr}
-1 & 0 & 0 \\
0 & -1 & 1 \\
0 & 1 & -1
\end{array}\right],\left[\begin{array}{ll}
0 & 0 \\
0 & 0
\end{array}\right],\left[\begin{array}{rrrr}
-1 & 1 & 0 & 0 \\
1 & -2 & 1 & 0 \\
0 & 1 & -2 & 1 \\
0 & 0 & 1 & -1
\end{array}\right]\right\} \text {, } \\
& G=\left[\begin{array}{rrrrrrrrr}
2 & -2 & 1 & -3 & 0 & 0 & 0 & 0 & 0 \\
-2 & 10 & -1 & 11 & 4 & 4 & 4 & 4 & 4 \\
1 & -1 & -3 & -5 & -4 & -1 & -1 & -1 & -1 \\
-3 & 11 & -5 & 9 & 4 & -1 & 3 & 3 & 3 \\
0 & 4 & -4 & 4 & 17 & -36 & 20 & -40 & -5 \\
0 & 4 & -1 & -1 & -36 & 75 & -70 & 92 & -53 \\
0 & 4 & -1 & 3 & 20 & -70 & 98 & -146 & 90 \\
0 & 4 & -1 & 3 & -40 & 92 & -146 & 178 & -118 \\
0 & 4 & -1 & 3 & -5 & -53 & 90 & -118 & 115
\end{array}\right] .
\end{aligned}
$$


It is easy to check that $H$ is negative definite and $G$ is indefinite. The matrix $M$ satisfies the condition of Theorem 5.1 of [8] from $H^{\infty}$-control problems. Hence, we can apply Algorithms $3.1,3.3$, and 3.6 to find an $M$-stable Lagrangian subspace $\mathcal{Y}_{\mathcal{L}}$. We first use Algorithm 3.1 to compute an orthonormal Jordan subbasis $Z[1: 14]$ corresponding to zero eigenvalues of $M^{2}$. Since zero eigenvalues of $M^{2}$ have partial multiplicities $\{1,1,2,2,4,4\}$, we check 2 -norms of the following matrices:

$$
\begin{array}{|l|c|c|c|c|} 
& M^{2} Z[1: 6] & M^{4} Z[7: 10] & M^{6} Z[11: 12] & M^{8} Z[13: 14] \\
\hline\|\cdot\|_{2} & 3.34 \mathrm{e}-14 & 1.72 \mathrm{e}-12 & 8.65 \mathrm{e}-12 & 1.79 \mathrm{e}-12 \\
\hline
\end{array}
$$

Next, we use Algorithm 3.3 to compute an orthonormal Jordan subbasis $Y[1: 14]$ corresponding to a zero eigenvalue of $M$. The zero eigenvalue of $M$ has partial multiplicities $\{2,4,8\}$; we check 2-norms of the following matrices:

\begin{tabular}{|l|l|l|l|l|}
\hline$\|\cdot\|_{2}$ & $\begin{array}{l}M Y[1: 3] \\
\|\end{array}$ & $M^{2} Y[4: 6]$ & $M^{3} Y[7: 8]$ & $M^{4} Y[9: 10]$ \\
\hline & $M^{5} Y[11]$ & $M^{6} Y[12]$ & $M^{7} Y[13]$ & $M^{8} Z[14]$ \\
\hline$\|\cdot\|_{2}$ & $2.25 \mathrm{e}-10$ & $4.22 \mathrm{e}-12$ & $7.29 \mathrm{e}-12$ & $2.31 \mathrm{e}-12$
\end{tabular}

Now, we compute the maximal isotropic subbasis $\Upsilon[1: 7]=\Upsilon_{s}$ of the stable Lagrangian subspace corresponding to zero eigenvalues. At the same time, the isotropicity of $\Upsilon[1: 7]$ is checked:

$$
\left\|\Upsilon[1: 7]^{T} J_{9} \Upsilon[1: 7]\right\|_{2}=1.96 \mathrm{e}-13 .
$$

Finally, we deflate the zero eigenvalue and the associated subbasis $\Upsilon[1: 7]$ of $M$ by using symplectic orthogonal transformations and get a $4 \times 4$ Hamiltonian matrix having eigenvalues $\{-1,-1,1,1\}$. Then we use algorithms of $[2,23,29]$ to find the rest subbasis $\Upsilon[8: 9]$ of the desired $M$-stable Lagrangian subspace $\mathcal{Y}_{\mathcal{L}}$. Consequently, a symmetric stable solution $X_{\text {sol }}$ of CARE (1.4) is computed by

$$
X_{\text {sol }}=-\Upsilon[10: 18,1: 9](\Upsilon[1: 9,1: 9])^{-1} .
$$

The 2-norm of the residual of the Riccati equation is $8.71 \mathrm{e}-14$.

6. Conclusions. In this paper, we have presented structure-preserving algorithms for computing an $M$-stable and an $(N, L)$-stable Lagrangian subspace of Hamiltonian matrices and symplectic pencils having purely imaginary and unimodular eigenvalues, respectively. These problems often arise in solving the continuous- or discrete-time $H^{\infty}$-optimal and linear-quadratic control problems, etc. The main approach of our algorithms is to find a maximal isotropic subbasis corresponding to each first half of Jordan blocks of purely imaginary eigenvalues (unimodular eigenvalues, respectively). Furthermore, we deflate the computed isotropic subbasis by using symplectic orthogonal transformations and get a reduced Hamiltonian matrix (symplectic pencil) having no purely imaginary (unimodular) eigenvalues. Then we compute the 
maximal stable isotropic subspace of the reduced Hamiltonian matrix (symplectic pencil) by applying some proposed methods of $[2,23,29]$. Thus, we obtain the desired stable Lagrangian subspace by combining these two computed isotropic subspaces. For the continuous case, we first compute an orthonormal Jordan basis corresponding to nonpositive eigenvalues of $M^{2}$ and then use it to determine the maximal isotropic Jordan subbasis corresponding to each first half of Jordan blocks of purely imaginary eigenvalues of $M$. The proposed algorithm is structure preserving and only uses orthogonal transformations. The dominant flops of the algorithm are in the step of reducing $M^{2}$ to a skew-Hamiltonian upper triangular matrix. It requires $O\left(n^{2}\right)$ flops for the deflation of the computed isotropic subbasis if the number of purely imaginary eigenvalues is of order 1 compared with the dimension of matrices. Numerical experiments performed on a number of constructive Hamiltonian matrices of dimension 30 with variant sizes of Jordan blocks have shown that our algorithm is stable and reliable in accuracy of the computed maximal isotropic subbasis. For the discretetime case, we also develop an algorithm to compute the maximal isotropic Jordan subbasis corresponding to each first half of Jordan blocks of unimodular eigenvalues of a symplectic pencil $N-\lambda L$. The approach is analogous to that developed in the continuous case by replacing the $M^{2}$-transformation by the $S+S^{-1}$-transformation of the symplectic pencil. The algorithm is structure preserving and uses orthogonal transformations but in the deflation step. Since the algorithm preserves the symplecticity for the pencil type, if the conditions of nonorthogonal transformations in the deflation step are fairly good, the proposed algorithm is still efficient and reliable.

\section{REFERENCES}

[1] B. D. O. Anderson and S. Vongpanitlerd, Network Analysis and Synthesis, Lecture Notes in Control and Information Science, Prentice-Hall, Englewood Cliffs, NJ, 1973.

[2] G. S. Ammar and V. Mehrmann, On Hamiltonian and symplectic Hessenberg forms, Linear Algebra Appl., 149 (1991), pp. 55-72.

[3] T. Beelen and P. Van Dooren, An improved algorithm for the computation of Kronecker's canonical form of a singular pencil, Linear Algebra Appl., 105 (1988), pp. 9-65.

[4] T. F. Chan, Rank revealing QR factorizations, Linear Algebra Appl., 88/89 (1987), pp. 67-82.

[5] D. Clements And K. Glover, Spectral transformations via Hermitian pencils, Linear Algebra Appl., 123 (1989), pp. 797-846.

[6] B. A. Francis AND J. C. Doyle, Linear control theory with an $H^{\infty}$-optimality criterion, SIAM J. Control Optim., 25 (1987), pp. 815-844.

[7] U. Flaschka, V. Mehrmann, and D. Zywietz, An analysis of structure preserving methods for symplectic eigenvalue problems, RAIRO Automat.-Prod. Inform. Ind., 25 (1991), pp. $165-190$.

[8] K. Glover, D. J. N. Limebeer, J. C. Doyle, E. M. Kasenally, and M. G. Safonov, A characterization of all solutions to the fourblock general distance problem, SIAM J. Control Optim., 39 (1991), pp. 283-324.

[9] G. Golub And C. F. VAN LoAn, Matrix Computation, The Johns Hopkins University Press, Baltimore, MD, 1983.

[10] P. IGLEsias, Robust and Adaptive Control for Discrete-Time Systems, Ph.D. dissertation, Department of Electrical Engineering, Cambridge University, U.K., 1991.

[11] V. Ionescu And M. Weiss, Two Riccati formulae for the discrete-time $H^{\infty}$-control problem, Internat. J. Control, 57 (1993), pp. 141-195.

[12] C. Kenney, A. J. Laub, and M. Wette, A stability-enhancing scaling procedure for SchurRiccati solvers, Systems Control Lett., 12 (1989), pp. 241-250.

[13] P. Lancaster, A. C. M. Ran, and L. Rodman, Hermitian solutions of the discrete algebraic Riccati equation, Internat. J. Control, 44 (1986), pp. 777-802.

[14] P. Lancaster and L. Rodman, Existence and uniqueness theorems for the algebraic Riccati equation, Internat. J. Control, 32 (1980), pp. 285-309.

[15] A. J. LAUB, A Schur method for solving algebraic Riccati equations, IEEE Trans. Automat. Control, 24 (1979), pp. 913-921. 
[16] A. J. Laub and K. Meyer, Canonical forms for symplectic and Hamiltonian Matrices, Celestial Mech., 9 (1974), pp. 213-238.

[17] W.-W. Lin, On reducing infinite eigenvalues of regular pencils by a nonequivalence transformation, Linear Algebra Appl., 78 (1986), pp. 207-231.

[18] W.-W. Lin, A new method for computing the closed loop eigenvalues of a discrete-time algebraic Riccati equation, Linear Algebra Appl., 96 (1987), pp. 157-180.

[19] L.-Z. Lu AND W.-W Lin, An iterative algorithm for the solution of the discrete-time algebraic Riccati equation, Linear Algebra Appl., 188/189 (1993), pp. 465-488.

[20] V. Mehrmann, The Autonomous Linear Quadratic Control Problem, Springer-Verlag, Berlin, 1991.

[21] C. Paige and C. F. Van Loan, A Schur decomposition for Hamiltonian matrices, Linear Algebra Appl., 41 (1981), pp. 11-32.

[22] R. V. PAtel, On computing the eigenvalues of a symplectic pencils, Linear Algebra Appl., 188 (1993), pp. 591-611.

[23] R. V. Patel, Z. Lin, And P. Misra, Computation of stable invariant subspaces of Hamiltonian matrices, SIAM J. Matrix Anal. Appl., 15 (1994), pp. 284-298.

[24] P. H. Petkov, N. D. Christov, and M. M. Konstantinov, On the numerical properties of the Schur approach for solving the matrix Riccati equation, Systems Control Lett., 9 (1987), pp. 197-201.

[25] G. W. Stewart and J.-G. Sun, Matrix Perturbation Theory, Academic Press, New York, 1990.

[26] A. J. Van Der Schaft and J. C. Willems, A new procedure for stochastic realization of spectral density matrices, SIAM J. Control Optim., 22 (1984), pp. 845-855.

[27] C. F. VAN LOAN, A symplectic method for approximating all the eigenvalues of a Hamiltonian matrix, Linear Algebra Appl., 61 (1984), pp. 233-251.

[28] J. C. Willems, Least squares stationary optimal control and the algebraic Riccati equations, IEEE Trans. Automat. Control, 16 (1971), pp. 621-634.

[29] H.-G. Xu, Solving Algebraic Riccati Equations via Skew-Hamiltonian Matrices, Ph.D. thesis, Department of Math, Fudan University, Shanghai, China, 1991. 